\title{
RIESZ DECOMPOSITIONS IN MARKOV PROCESS THEORY
}

\author{
$\mathrm{BY}$ \\ R. K. GETOOR ${ }^{1}$ AND J. GLOVER ${ }^{2}$
}

\begin{abstract}
Riesz decompositions of excessive measures and excessive functions are obtained by probabilistic methods without regularity assumptions. The decomposition of excessive measures is given for Borel right processes. The results for excessive functions are formulated within the framework of weak duality. These results extend and generalize the pioneering work of Hunt in this area.
\end{abstract}

1. Introduction. Since the appearance of Hunt's pioneering work [6], it has been clear that there is an identity between certain aspects of the theory of Markov processes and potential theory. In Hunt's work, the processes that were emphasized are what are now called Hunt processes. (He also considered a class of subprocesses of a Hunt process.) In proving many of his results, Hunt assumed certain additional regularity hypotheses about his processes. Not surprisingly, the coarser potential theoretic results needed few hypotheses beyond the basic requirement that the process under study be a Hunt process, while the more delicate results needed additional regularity hypotheses such as the existence of a dual process or that the resolvent or dual resolvent be Feller or strong Feller. It slowly became clear that it was necessary to remove many of these regularity hypotheses in order to advance the theory. This led to the introduction of right processes by Meyer. However, it seems that the study of the probabilistic aspects of these processes has outstripped to some degree the study of their associated potential theory. The present paper is devoted largely to the study of the potential theory of right processes and, in particular, to the Riesz decompositions of excessive measures and excessive functions. Since analytic methods are not directly available, it is necessary to use probabilistic techniques in this study. Our main tools are appropriate versions of Ray-Knight compactification theory, time reversal and Revuz measures.

The contents of this paper were inspired in part by Hunt's original work and in part by the excellent set of Paris lecture notes on duality of Markov processes written by J. B. Walsh [11]. As we describe the contents of the paper, we shall recall some of their results to illustrate the historical framework into which our results fit.

Let $X=\left(\Omega, \mp, \digamma_{t}, X_{t}, \theta_{t}, P^{x}\right)$ be a right process on a Lusin topological state space $E$ with Borel field $\mathcal{E}$. A point $\Delta \notin E$ will serve as cemetery point. Let $P_{t}$ and $U^{\alpha}$ denote the semigroup and resolvent of $X$. If $U^{\alpha} f$ is $\mathcal{E}$-measurable

Received by the editors August 25, 1983.

1980 Mathematics Subject Classification. Primary 60J45, 60J25.

${ }^{1}$ Research supported in part by NSF Grant MCS 79-23922.

${ }^{2}$ Research supported by an NSF Postdoctoral Research Fellowship in the Mathematical Sciences. 
whenever $f$ is a positive $\mathcal{E}$-measurable function, then we say that $X$ is a Borel right process. In fact, a Borel right process on a Lusin state space $E$ is nothing but a right continuous, strong Markov process with no branch points and with a Borel measurable resolvent. The process is said to be transient if there is a strictly positive $\mathcal{E}$-measurable function $q$ so that $U q \leq 1$. We restrict our attention to transient Borel right processes throughout this paper. See [3] for some apparently weaker conditions that are equivalent to this definition of transience.

$\S 2$ is devoted to studying the Riesz decomposition of excessive measures. Hunt assumed a form of transience (his hypothesis (E)), a Feller condition (his hypothesis (C)) and the fact that $E$ is locally compact with a countable base (LCCB) in studying excessive measures of a Hunt process. He showed that an excessive measure $\xi$ can be decomposed into the sum of a potential piece $\pi$ and a "harmonic" piece $\eta$, each of which can be characterized by means of a balayage operator on excessive measures. Using compactification methods, we demonstrate that his decomposition can be extended to excessive measures of a transient Borel right process ((2.2), (2.8)). We also show how to characterize $\pi$ and $\eta$ in the strong order on excessive measures (2.10).

Thus, studying excessive measures seems to require little in the way of regularity hypotheses, but studying excessive functions often requires more. One hypothesis used extensively in earlier work is that of strong duality. We outline the major ideas here for later use. Let $\hat{X}=\left(\hat{\Omega}, \hat{\mathcal{F}}, \hat{\mathcal{F}}_{t}, \hat{X}_{t}, \hat{\theta}_{t}, \hat{P}^{x}\right)$ be another transient Borel right process on $(E, \mathcal{E})$ with semigroup $\hat{P}_{t}$ and resolvent $\hat{U}^{\alpha}$, and let $m$ be a fixed $\sigma$-finite measure. If

$$
\int_{E} P_{t} f \cdot g d m=\int_{E} f \cdot \hat{P}_{t} g d m
$$

for all positive $\mathcal{E}$-measurable functions $f$ and $g$, and if

$$
\left.X_{t-} \text { exists in } E \text { for all } t \in\right] 0, \zeta\left[, \quad \hat{X}_{t-} \text { exists in } E \text { for all } t \in\right] 0, \hat{\zeta}[\text {, }
$$

then $X$ and $\hat{X}$ are said to be in weak duality with respect to $m$ (or the triple $(X, \hat{X}, m)$ is in weak duality). If, in addition, for every $\alpha \geq 0$ and for every $x$ in $E$,

$$
U^{\alpha}(x, \cdot) \ll m \text { and } \hat{U}^{\alpha}(x, \cdot) \ll m,
$$

then $X$ and $\hat{X}$ are said to be in strong duality with respect to $m((X, \hat{X}, m)$ is in strong duality). In [9], Walsh has shown that under (1.1), (1.2) holds almost surely $P^{x}$ for $m$ almost all $x$, and it follows that under (1.1) and (1.3), (1.2) holds almost surely $P^{x}$ for all $x$. If $(X, \hat{X}, m)$ is in strong duality, then for each $\alpha \geq 0$, a potential density $u^{\alpha}(x, y)$ can be chosen which is $\mathcal{E} \times \mathcal{E}$-measurable and so that

(i) $U^{\alpha}(x, d y)=u^{\alpha}(x, y) m(d y)$

$$
\hat{U}^{\alpha}(x, d y)=u^{\alpha}(y, x) m(d y)
$$

(ii) $x \mapsto u^{\alpha}(x, y)$ is $\alpha$-excessive for $X$,

$$
y \mapsto u^{\alpha}(x, y) \quad \text { is } \alpha \text {-excessive for } \hat{X} \text {. }
$$

As mentioned above, strong duality has been used extensively, and a discussion of standard processes in strong duality may be found in Chapter VI of [1]. The systematic study of weak duality is more recent and may be found in [5]. Part of 
the purpose of this paper is to show that weak duality is an appropriate framework for potential theory, despite the lack of potential densities $u^{\alpha}(x, y)$. Several of the results of this paper are new even for standard processes in strong duality (since precursors of our results were often discussed assuming strong duality and various Feller hypotheses on LCCB state spaces).

$\S 3$ contains elementary results about weak duality which will be needed in later sections. In strong duality, one can use the potential density $u(x, y)=u^{0}(x, y)$ to define the potential of a positive measure $\mu$ by setting

$$
U \mu(x)=\int_{E} u(x, y) \mu(d y) .
$$

By Fubini's theorem, $U \mu(x) m(d x)=\mu \hat{U}(d x)$. It is this relationship which is useful in extending the notion of "potential of a measure" to weak duality, where potential densities $u^{\alpha}(x, y)$ may not exist. Let $S$ denote the class of Borel measurable excessive functions $u$ of $X$ which are finite a.e. $m$. A function $u$ in $S$ will be called the potential of a measure $\mu$ provided $u m=\mu \hat{U}$, and we write $u=U(\mu)$. Note that $u$ is determined only $m$ a.e. in general. Let $\mathcal{M}$ denote the class of measures $\mu$ so that $\mu \hat{U}=u m$ with $u$ in $S$. It turns out that $\mathcal{M}$ is the class of measures $\mu$ on $E$ which do not charge cofinely open $m$-copolar sets and so that $\mu \hat{U}$ is $\sigma$-finite (3.8). A rich potential theory can be constructed in weak duality by restricting attention to potentials $U(\mu)$ of measures $\mu$ in $\mathcal{M}$. For example (3.9), if $\mu \in \mathcal{M}$ and $v \in S$ with $v \leq U(\mu)$ a.e. $m$, then $v=U(\nu)$ a.e. $m$ for some measure $\nu \in \mathcal{M}$. In the case of transient Borel right processes in strong duality, the only cofinely open $m$-copolar set is the null set, $\mathcal{M}$ is the class of all measures $\mu$ on $E$ with $\mu \hat{U} \sigma$-finite, and $U(\mu)$ is determined everywhere on $E$ and is equal to $U \mu$ defined in (1.5), since excessive functions which agree $m$ a.e. are, in fact, identical.

The results of $\S 2$ are used in $\S 4$ to obtain a Riesz decomposition theorem for excessive functions of transient Borel right processes in weak duality (4.5). That is, an excessive function $u \in S$ is decomposed into a sum of a potential $U(\mu)$ of a measure $\mu$ in $\mathcal{M}$ and a "harmonic" function $h$. In Chapter VI of [1], a Riesz decomposition of $u$ into a sum $u=U \mu+h$ is given under the assumption that $E$ is LCCB, $X$ and $\hat{X}$ are standard processes in strong duality, and $\left(\hat{U}^{\alpha}\right)_{\alpha \geq 0}$ is strong Feller. There, $U \mu$ and $h$ are characterized in terms of balayage. We give a similar characterization in (4.2) and (4.4), but consult the discussion before (4.2) carefully for details about the set $\mathcal{K}(\hat{E})$ used in the balayage characterization. An analogue of the strong order for excessive functions which is appropriate for weak duality is defined as follows: an excessive function $w$ strongly $m$-dominates an excessive function $v$ if there is an excessive function $p$ with $w=v+p$ a.e. $m$. (In strong duality, the strong $m$-domination order agrees with the usual strong order.) In (4.8), we characterize $U(\mu)$ and $h$ in the decomposition of $u$ above in terms of the strong $m$-domination order. As far as we know, the results in $\S 4$ are new even when specialized to the case of standard processes in strong duality.

$\S 5$ contains several well-known facts about $h$-transforms drawn from [10 and 11]. Before continuing, we briefly recall the basic notation for $h$-transforms. See §5. If $u \in S$, then $P_{t}^{u} f(x)=u(x)^{-1} P_{t}(u f)(x)$ defines a semigroup on $E_{u}=\{0<$ $u<\infty\}$, and we let $\left(X_{t}, P^{x / u}\right)$ denote the Borel right process with semigroup $\left(P_{t}^{u}\right)$ constructed on canonical path space. In $\S 5$, we give a version of Nagasawa's 
theorem which is valid for processes $(X, \hat{X}, m)$ in weak duality. Let $\tilde{X}$ be the right continuous reversal of $X$ from $\zeta$-the precise definition is given in $\S 5$. If $\mu \in \hat{\mathcal{M}}$ (the class $\mathcal{M}$ defined above, but relative to $\hat{X}$ ) and $u=\hat{U}(\mu)$, then Nagasawa's theorem tells us that $\left(\tilde{X}_{t}, P^{\mu}\right)$ for $t>0$ is a time homogeneous Markov process with semigroup $\left(\hat{P}_{t}^{u}\right)$, but it does not give any information about the entrance law of $\tilde{X}_{t}$, in general. The main result (6.5) of $\S 6$ is an extension of Nagasawa's theorem that identifies this entrance law under appropriate conditions. Theorem (6.5) seems to be of considerable probabilistic interest, and its Corollary (6.10) is fundamental in the applications to the potential theory in $\S 7$.

In his Paris lecture notes, Walsh considered the following situation: $X$ and $\hat{X}$ are transient Borel right processes (actually, he permits branch points) in strong duality with respect to $m$ so that:

(i) for every $\alpha \geq 0$ and for every bounded positive $\mathcal{E}$-measurable function $f, \hat{U}^{\alpha} f$ is continuous on $E$;

(ii) $\hat{U} 1(x)$ is bounded in $x$.

Walsh proved in his Proposition (4.3) that $u$ is the potential $U \mu$ of a measure $\mu$ if and only if

$$
P^{x / u}\left[X_{\varsigma-} \text { exists in } E, 0<\varsigma<\infty\right]=1
$$

for every $x$ in $E_{u}$. In $\S 7$, we extend his result to the case of Borel right processes in weak duality. Our methods are quite different from his. Choose $q$ with $0<q \leq$ $1, m(q)<\infty$ and $h \equiv \hat{U} q \leq 1$. Fix a countable set $\left(g_{n}\right)$ which is dense in the set of bounded positive uniformly continuous functions on $E$, and define

$$
\begin{array}{r}
\Lambda=\bigcap_{r}\left\{\begin{array}{l}
n \\
n
\end{array} 0<\infty, X_{\zeta-} \text { exists in } E, h\left(X_{\zeta-}\right)=h\left(X_{\zeta}\right)_{-},\right. \\
\left.\hat{U}^{r}\left(h g_{n}\right)\left(X_{\zeta^{-}}\right)=\hat{U}^{r}\left(h g_{n}\right)\left(X_{\zeta}\right)_{-}\right\},
\end{array}
$$

where $r$ ranges over the positive rationals. It is immediate, under Walsh's conditions given above, that almost surely, $\Lambda=\left\{X_{\zeta-}\right.$ exists in $\left.E, 0<\varsigma<\infty\right\}$. The main result of $\S 7$ is that $u \in S$ is the potential $U(\mu)$ of a measure $\mu \in \mathcal{M}$ if and only if $P^{x / u}(\Lambda)=1 m$ a.e. on $E_{u}$. Moreover, for any $u \in S, u P^{\cdot / u}(\Lambda)$ is the potential of a measure in $\mathcal{M}$ and

$$
u=u P^{\cdot / u}(\Lambda)+u P^{\cdot / u}\left(\Lambda^{c}\right)
$$

is the Riesz decomposition $u=U(\mu)+h$ of $\S 4$ described earlier. The main tools in proving these results are (6.10) and an extension of the Revuz formula of [5] to additive functionals which may jump at $\zeta$. This extension is discussed in the Appendix and should prove to be useful in other situations.

NotATION. Most of the notation we use is standard and can be found in $[\mathbf{1}]$ and [2], with one glaring exception which we shall discuss later. We shall use the symbol $\equiv$ to mean "is defined to be". If $(W, W)$ is any measurable space, then $\mathcal{W}$ (resp. $b \mathcal{W}, \mathcal{W}^{+}, b W^{+}$) will also be the collection of $\mathcal{W}$-measurable numerical functions on $W$ (resp. which are bounded, which are positive, which are bounded and positive). If $D$ is any metric space, $\mathbf{C}_{u}(D)$ (resp. $b \mathbf{C}_{u}(D)$ ) is the collection of uniformly continuous functions on $D$ (resp. which are bounded). The glaring exception mentioned above is the following: The semigroup and resolvent of $X$ are $P_{t}(x, d y)$ and $U^{\alpha}(x, d y)$ so that $P_{t} f(x)=\int f(y) P_{t}(x, d y)$ and $U^{\alpha} f(x)=\int f(y) U^{\alpha}(x, d y)$. Similarly, the semigroup and resolvent of $\hat{X}$ are $\hat{P}_{t}(x, d y)$ and $\hat{U}^{\alpha}(x, d y)$ so that $\hat{P}_{t} f(x)=$ 
$\int f(y) \hat{P}_{t}(x, d y), \hat{U}^{\alpha} f(x)=\int f(y) \hat{U}^{\alpha}(x, d y)$ and $\mu \hat{U}^{\alpha}(\cdot)=\int \mu(d x) \hat{U}^{\alpha}(x, \cdot)$. This is not the notation used in $[\mathbf{1}]$ and $[\mathbf{6}]$ for $\hat{P}_{t}$ and $\hat{U}^{\alpha}$. The present notation is better suited to weak duality. As usual, $P_{t}^{\alpha} \equiv e^{-\alpha t} P_{t}$ and $\hat{P}_{t}^{\alpha} \equiv e^{-\alpha t} \hat{P}_{t}$. If $f \in \mathcal{E}^{+}$and $\nu$ is a measure on $E$, then $f \nu$ denotes the measure $f(x) \nu(d x)$, while $\nu f=\nu(f)=\int f d \nu$. In more complicated formulas, we shall sometimes write $f \cdot \nu$ or $\nu \cdot f$ for clarity in place of $f \nu$. For example, $\mu \hat{U} \cdot g$ is the measure $A \rightarrow \int_{A} \mu \hat{U}(d x) g(x)$, while $\mu \hat{U} g=\int \mu \hat{U}(d x) g(x)$.

2. Excessive measures. Let $X=\left(\Omega, \mathcal{F}, \mathcal{F}_{t}, X_{t}, \theta_{t}, P^{x}\right)$ be a Borel right process on a Lusin topological state space $(E, \mathcal{E})$ with semigroup $P_{t}$ and resolvent $U^{\alpha}$ and with cemetery point $\Delta \notin E$. No duality hypotheses are assumed in this section, but we do assume that $X$ is transient in the following sense: there is a function $q \in \mathcal{E}$ with $0<q \leq 1$ and $U q \leq 1$. Most of the results in this section extend to the case of arbitrary (universally measurable) right processes on $U$-spaces, but we restrict ourselves to the case of Borel right processes since subsequent sections will be concerned only with that case.

(2.1) Definition. A measure $\xi$ is $\alpha$-excessive for $X$ (or $U$ ) provided $\xi$ is $\sigma$-finite and $\xi P_{t}^{\alpha} \leq \xi$ for every $t \geq 0$. The class of $\alpha$-excessive measures will be denoted by $\operatorname{Exc}^{\alpha}(U)$.

Excessive measures of Borel right processes are investigated in [4], to which we shall often refer. In order to discuss the Riesz decomposition of excessive measures in the present situation we extend the idea of the proof of $[4,(4.1)]$. To do this, we shall recall the extension of the Ray-Knight procedure discussed in $\S 3$ of $[4]$; the reader is referred there for further details. Given a function $q$ as described in the first paragraph of this section, we set $V g(x)=U q(x)^{-1} U(q g)(x)$, so that $V$ is the zero-potential of a process $Y=\left(\Omega, \mathcal{G}, \mathcal{G}_{t}, Y_{t}, \theta_{t}, Q^{x}\right)$ which can be obtained as follows: produce a process $Z$ by $h$-transforming $X$ with $U q$, then produce $Y$ by time-changing $Z$ by the right continuous inverse of the additive functional $A_{t}=$ $\int_{0}^{t}(q / U q)\left(Z_{s}\right) d s$. Since $q \in \mathcal{E}, V^{\alpha} g(x) \in \mathcal{E}$ for every $g \in \mathcal{E}^{+}$, so $Y$ is a Borel right process. Let $Q_{t}$ be the semigroup of $Y_{t}$. Since $V 1_{E}=1_{E}, R_{t}=e^{t} Q_{t}$ is a Markovian semigroup on $E$. If we set

$$
W^{\alpha} g(x)=\int_{0}^{\infty} e^{-\alpha t} R_{t} g(x) d t
$$

then $W^{1+\alpha}=V^{\alpha}$. Let $\mathbf{R}$ denote the Ray cone generated by $W^{\alpha}$. If $\bar{E}$ is the Ray compactification of $E$ constructed with respect to $\mathbf{R}$, then $E$ is Borel in $\bar{E}$. If $\mathbf{C}_{u}(\bar{E})$ denotes the continuous functions on $\bar{E}$, then $W^{\alpha}$ extends to a resolvent $\bar{W}^{\alpha}$ on $\bar{E}$, and $\bar{W}^{\alpha}: \mathbf{C}_{u}(\bar{E}) \rightarrow \mathbf{C}_{u}(\bar{E})$. Let $\bar{V}^{\alpha}=\bar{W}^{1+\alpha}$, and let $\bar{Y}=\left(\bar{Y}_{t}, \bar{Q}^{x}\right)$ be the Ray process on $\bar{E}$ with resolvent $\bar{V}^{\alpha}$ and semigroup $\bar{Q}_{t}$. Let $\bar{\sigma}=\inf \left\{t\right.$ : $\left.\bar{Y}_{t} \notin E\right\}$. If $x \in E$, then $\left(Y_{t}, Q^{x}\right)$ and $\left(\bar{Y}_{t}, \bar{\sigma}, \bar{Q}^{x}\right)$ are indistinguishable in a sense made precise in $[2,(11.8)]$. Of course, $\left(\bar{Y}_{t}, \bar{\sigma}\right)$ is the process $\bar{Y}_{t}$ killed at $\bar{\sigma}$. For simplicity we may use the same cemetery point $\Delta \notin \bar{E}$ for both $\left(Y_{t}\right)$ and $\left(\bar{Y}_{t}, \bar{\sigma}\right)$. Let $E^{r}$ denote the set of regular points for $E$ in $\bar{E}$ (relative to $\bar{Y}$ ), and let $D$ be the set of nonbranch points for $\bar{Y}$ in $\bar{E}$. Set $F=D \cap E^{r}$. Then $E \subset F$.

(2.2) Proposition. Let $\xi \in \operatorname{Exc}(U)$, and let $q$ be chosen as above and so that $\xi(q)<\infty$. There are a measure $\rho$ on $E$ and a measure $\gamma$ on $F-E$ so that $\xi=\rho U+\gamma \bar{V} \cdot q^{-1}$. Moreover, $\rho$ and $\gamma$ are unique. 
REMARK. It is worth keeping in mind throughout this discussion that $\bar{E}=$ $\bar{E}(q)$ depends on $q$. Thus, for each $\xi \in \operatorname{Exc}(U)$, we may have to pick a different compactification $\bar{E}(q)$ to obtain the result. Of course, once $q$ is fixed, $\bar{E}(q)$ will work for all $\xi \in \operatorname{Exc}(U)$ with $\xi(q)<\infty$.

Proof. Choose measures $\mu_{n}$ on $E$ with $\mu_{n} U$ increasing to $\xi[4,(1.5)]$. If $h=U q$, then $\left(h \mu_{n}\right) V$ increases to $q \xi$, so $q \xi \in \operatorname{Exc}(V)$. Now $\left(h \mu_{n}\right) \bar{V} 1=\left(h \mu_{n}\right) V 1 \leq$ $\xi(q)<\infty$. Since $\bar{V}^{\alpha} 1=(\alpha+1)^{-1}$ on $\bar{E},\left(h \mu_{n}\right)$ has a subsequence $\left(h \mu_{n_{k}}\right)$ converging weakly to a measure $\bar{\lambda}$ on $\bar{E}$. If $\bar{f}$ is in $\overline{\mathbf{R}}$ (the extension of the Ray cone $\mathbf{R}$ to $\bar{E}$ ), then

$$
\bar{\lambda} \bar{V} \bar{f}=\lim _{k}\left(h \mu_{n_{k}}\right) \bar{V} \bar{f}=\xi(q \bar{f}),
$$

so $\bar{\lambda} \bar{V}=q \xi$ since $\overline{\mathbf{R}}-\overline{\mathbf{R}}$ is dense in $\mathbf{C}_{u}(\bar{E})$. If we set $\lambda=\bar{\lambda} \bar{Q}_{0}$, then $\lambda \bar{V}=q \xi$. Since $q \xi$ is concentrated on $E, \lambda$ must be concentrated on $F$. We have

$$
q \xi=\left(1_{E} \lambda\right) \bar{V}+\left(1_{F-E} \lambda\right) \bar{V}=\left(h^{-1} 1_{E} \lambda\right) U \cdot q+\left(1_{F-E} \lambda\right) \bar{V}
$$

or

$$
\xi=\left(h^{-1} 1_{E} \lambda\right) U+\left(1_{F-E} \lambda\right) \bar{V} \cdot q^{-1} .
$$

Set $\rho=\left(h^{-1} 1_{E}\right) \lambda$ and $\gamma=1_{F-E} \lambda$. Suppose $\rho^{\prime}$ is a measure on $E$ and $\gamma^{\prime}$ is a measure on $F-E$ so that $\xi=\rho^{\prime} U+\gamma^{\prime} \bar{V} \cdot q^{-1}$. Then $h\left(\rho^{\prime}-\rho\right) \bar{V}=\left(\gamma^{\prime}-\gamma\right) \bar{V}$. Since $Y$ is a right process when restricted to $F$ and $\rho^{\prime}-\rho$ and $\gamma^{\prime}-\gamma$ are carried by $F, h\left(\rho^{\prime}-\rho\right)=\gamma^{\prime}-\gamma$ by $[\mathbf{4},(1.1)]$. Since $\rho^{\prime}-\rho$ is carried by $E, h$ is strictly positive on $E$, and $\gamma^{\prime}-\gamma$ is carried by $F-E, \rho^{\prime}=\rho$ and $\gamma^{\prime}=\gamma$. Q.E.D.

(2.4) DEFInITION. A measure $\xi \in \operatorname{Exc}^{\alpha}(U)$ is said to be an $\alpha$-measure potential if $\xi=\mu U^{\alpha}$ for some measure $\mu$ on $E$. The class of $\alpha$-measure potentials is denoted by $\operatorname{Pot}^{\alpha}(U)$.

It is natural to expect the measure $\gamma \bar{V} \cdot q^{-1}$ in (2.2) to be "harmonic" since $\gamma$ is carried by the "boundary" $F-E$. One may well wonder whether or not the property of being harmonic depends upon the choice of $q$ and hence the choice of compactification $\bar{E}=\bar{E}(q)$. It does not (see (2.10)), so we make the following

(2.5) Definition. Let $\xi \in \operatorname{Exc}(U)$. If for some $q$ chosen as in $(2.2), \xi=\gamma \bar{V} \cdot q^{-1}$ for some measure $\gamma$ carried by $F-E$, then $\xi$ is said to be harmonic. The class of harmonic measures is denoted by $\operatorname{Har}(U)$.

We now investigate alternative characterizations of measures in $\operatorname{Pot}(U)$ and $\operatorname{Har}(U)$. Assuming his hypotheses $(\mathrm{C})$ and $(\mathrm{E})$, Hunt characterized measures in $\operatorname{Pot}(U)$ and $\operatorname{Har}(U)$ in terms of balayage of measures [6, (14.7), (14.6)].

(2.6) Definition. Let $\xi \in \operatorname{Exc}(U)$, and let $B$ be a nearly Borel set. Choose measures $\mu_{n}$ on $E$ so that $\mu_{n} U$ increases to $\xi$ and define the balayage operator

$$
L_{B} \xi=\lim _{n} \mu_{n} P_{B} U \text {. }
$$

We refer the reader to $\S \S 8$ and 14 of $[6]$ for the following properties of $L_{B}$. Also $\S 1$ of [4] is useful for the straightforward extension of Hunt's results to right processes.

(i) $L_{B} \xi$ is independent of the choice of sequence $\left(\mu_{n}\right)$;

(ii) $L_{B} \xi \leq \xi, L_{B} \xi=\xi$ on $B$, and $L_{B} \xi \in \operatorname{Exc}(U)$;

(iii) if $B$ is finely open, then $L_{B} \xi=\inf \{\lambda \in \operatorname{Exc}(U): \lambda \geq \xi$ on $B\}$;

(iv) if $\eta \in \operatorname{Exc}(U)$, then $L_{B}(\xi+\eta)=L_{B}(\xi)+L_{B}(\eta)$;

(v) $L_{B}(\mu U)=\mu P_{B} U$.

(2.7) Definition. If $\tilde{E}$ is any compactification of $E, \mathcal{K}(\tilde{E})=\{K: K$ is compact in $\tilde{E} ; K \subset E\}$. If $K \in \mathcal{K}(\tilde{E})$, then $K^{c}$ means $E-K$. 
(2.8) Theorem. Let $\xi \in \operatorname{Exc}(U)$ and choose $q$ as in Proposition (2.2). Let $\bar{E}$ be the compactification described above (2.2). Then $\xi \in \operatorname{Pot}(U)$ if and only if $\inf \left\{L_{K^{c}} \xi: K \in K(\bar{E})\right\}=0$, and $\xi \in \operatorname{Har}(U)$ if and only if $L_{K^{c}} \xi=\xi$ for every $K \in \mathcal{K}(\bar{E})$.

REMARK. Recall that $\mathcal{E}$ is the trace on $E$ of the Borel field of $\bar{E}[\mathbf{2},(11.3)]$, so that $K$ and $K^{c}$ are in $\mathcal{E}$. Thus $L_{K^{c}} \xi$ is well defined by (2.6).

Before proving this, let us see what this says in the "classical cases;" i.e., assume $\left(U^{\alpha}\right)_{\alpha \geq 0}$ is strong Feller and $E$ is locally compact with a countable base. Then $q$ may be chosen so that $U q$ is bounded and continuous on $E$. In this case $\mathcal{K}(\bar{E})$ is the collection of sets in $E$ which are compact in $E$. This should be compared with $[6,(14.6),(14.7)]$.

PROOF. Fix $\xi \in \operatorname{Exc}(U)$ and $q$ as in (2.2) so that $\xi=\rho U+\gamma \bar{V} \cdot q^{-1}$, with $\rho$ a measure on $E$ and $\gamma$ a measure on $F-E$. For any $\nu \in \operatorname{Exc}(V)$, and for any sequence $\mu_{n}$ with $\mu_{n} V$ increasing to $\nu$, we set $M_{B} \nu=\lim _{n} \mu_{n} Q_{B} V$, so that $M_{B}$ is the balayage operator for the process $Y$ defined above (2.2). Since a measure $\lambda$ is in $\operatorname{Exc}(U)$ if and only if $q \lambda \in \operatorname{Exc}(V)$, it follows from (2.6)(iii) that $L_{B}=q^{-1} M_{B} q$ for any set $B \in \mathcal{E}$ which is finely open for $X$ (and hence is also finely open for $Y$ ). Thus, since $q \xi=(h \rho) V+\gamma \bar{V}$,

$$
\inf \left\{L_{K^{c}} \xi: K \in \mathcal{K}(\bar{E})\right\}=q^{-1} \inf \left\{M_{K^{c}}((h \rho) V+\gamma \bar{V}): K \in \mathcal{K}(\bar{E})\right\} .
$$

Fix for the moment $K \in \mathcal{K}(\bar{E})$. Applying the comments following (2.6), we see that $M_{K^{c}}(h \rho V+\gamma \bar{V})=(h \rho) Q_{K^{c}} V+M_{K^{c}}(\gamma \bar{V})$. Because $K^{c}$ is finely open for $Y, M_{K^{c}}(\gamma \bar{V})=\inf \left\{\lambda \in \operatorname{Exc}(V): \lambda \geq \gamma \bar{V}\right.$ on $\left.K^{c}\right\}$. If $\operatorname{Exc}(\bar{V} \mid F)$ denotes the class of excessive measures for the (right) process $\bar{Y}^{F}(\bar{Y}$ restricted to $F)$, then $\bar{\lambda} \in \operatorname{Exc}(\bar{V} \mid F)$ if and only if $\bar{\lambda} \in \operatorname{Exc}(V)$. (Note that $\bar{\lambda} \in \operatorname{Exc}(\bar{V} \mid F)$ implies $\bar{\lambda}$ is carried by $E$ and recall $F-E$ is polar for $\bar{Y}^{F}$.) Therefore

$$
M_{K^{c}}(\gamma \bar{V})=\inf \{\bar{\lambda} \in \operatorname{Exc}(\bar{V} \mid F): \bar{\lambda} \geq \gamma \bar{V} \text { on } F-K\},
$$

since both $\bar{\lambda}$ and $\gamma \bar{V}$ are carried by $E$. Applying (2.6)(iii) to $\bar{Y}^{F}$, we obtain $M_{K^{c}}(\gamma \bar{V})=\gamma \bar{Q}_{F-K} \bar{V}$. But $\gamma$ is carried by $F-E \subset F-K$ and $F-K$ is finely open for $\bar{Y}^{F}$. As a result $\gamma \bar{Q}_{F-K}=\gamma$ and so $M_{K^{c}}(\gamma \bar{V})=\gamma \bar{V}$ for each $K \in \mathcal{K}(\bar{E})$.

Now choose $H_{n}$ open in $\bar{E}, H_{n} \supset \bar{E}-E$ so that $T_{H_{n}}$ increases to $\bar{\sigma}=T_{\bar{E}-E}$, a.s. $\bar{Q}^{\rho}$. Let $K_{n}=\bar{E}-H_{n}$. Then $K_{n} \in \mathcal{K}(\bar{E})$, and since $\rho$ is carried by $E$ and $K_{n}^{c} \subset H_{n}$

$$
(h \rho) Q_{K_{n}^{c}} V f=\bar{Q}^{h \rho} \int_{T_{K_{n}^{c}}}^{\bar{\sigma}} f\left(\bar{Y}_{t}\right) d t \leq \bar{Q}^{h \rho} \int_{T_{H_{n}}}^{\bar{\sigma}} f\left(\bar{Y}_{t}\right) d t
$$

for $f \in b \mathcal{E}^{+}$. This last expression approaches zero as $n$ approaches infinity and so $h \rho Q_{K_{n}^{c}} V$ decreases to zero. Combining these results gives

$$
\inf \left\{L_{K^{c}} \xi: K \in \mathcal{K}(\bar{E})\right\}=q^{-1} \inf \left\{M_{K^{c}} q \xi: K \in \mathcal{K}(\bar{E})\right\}=\gamma \bar{V} \cdot q^{-1} .
$$

By the uniqueness part of (2.2), $\xi \in \operatorname{Pot}(U)$ if and only if $\gamma=0$, and $\xi \in \operatorname{Har}(U)$ if and only if $\rho=0$. The result follows. Q.E.D.

Now we turn to an examination of the strong ordering of excessive measures. 
(2.9) Definition. $\xi \in \operatorname{Exc}(U)$ is said to strongly dominate $\lambda \in \operatorname{Exc}(U)$ if there exists $\mu \in \operatorname{Exc}(U)$ with $\xi=\lambda+\mu$.

There is a closely related notion of strong domination of excessive functions (see $\S 4)$, and in fact the next few results will be used there. Given $\xi \in \operatorname{Exc}(U)$, we showed in (2.2) that $\xi$ can be decomposed as $\rho U+\gamma \bar{V} \cdot q^{-1}=\rho U+\eta$. The next result characterizes $\rho U$ and $\eta$ without reference to a compactification (so that our definition of the class $\operatorname{Har}(U)$ does not depend on the compactification chosen).

(2.10) THEOREM. If $\xi \in \operatorname{Exc}(U)$, choose $q$ as in (2.2) and write $\xi=\rho U+$ $\gamma \bar{V} \cdot q^{-1}=\rho U+\eta$. Then:

(i) $\eta$ is the largest excessive measure dominated by $\xi$ which strongly dominates no nonzero measure in $\operatorname{Pot}(U)$.

(ii) $\eta$ is the largest excessive measure in the strong order which is strongly dominated by $\xi$ and which strongly dominates no nonzero measure in $\operatorname{Pot}(U)$.

(iii) $\rho U$ is the largest measure (in either the ordinary order or the strong order) in $\operatorname{Pot}(U)$ which is strongly dominated by $\xi$.

ProOF. (i) Suppose $\pi \in \operatorname{Exc}(U)$ and $\pi \leq \xi$. Then (in the notation of (2.2) (so $h=U q)$ ), there is a measure $\mu$ on $F$ so that $q \pi=\mu \bar{V}$. It is easy to check that such a measure $\pi$ strongly dominates no nonzero measure in $\operatorname{Pot}(U)$ if and only if $\mu$ is carried by $F-E$. In this case, we have $(h \rho) \bar{V}+\gamma \bar{V} \geq \mu \bar{V}$ with $\rho$ carried by $E$, and $\gamma$ and $\mu$ carried by $F-E$. Thus we may choose a decreasing sequence $\left(G_{n}\right)$ of open sets in $\bar{E}$ containing $\bar{E}-E$ so that $T_{G_{n}}$ increases to $T_{\bar{E}-E}$ a.s. $\bar{Q}^{\rho}$. Then $(h \rho) \bar{Q}_{G_{n}} \bar{V}+\gamma \bar{Q}_{G_{n}} \bar{V} \geq \mu \bar{Q}_{G_{n}} \bar{V}$. (Here we have used the fact that if $\lambda \bar{V} \leq \nu \bar{V}$ and $B$ is Borel, then $\lambda \bar{Q}_{B} \bar{V} \leq \nu \bar{Q}_{B} \bar{V}$-given $f \geq 0$, choose $\left(f_{n}\right)$ with $\bar{V} f_{n}$ increasing to $\bar{Q}_{B} \bar{V} f$.) But $\gamma \bar{Q}_{G_{n}}=\gamma$ and $\mu \bar{Q}_{G_{n}}=\mu$ since $\gamma$ and $\mu$ are carried by $F-E \subset G_{n}$, and $(h \rho) \bar{Q}_{G_{n}} \bar{V}=(h \rho) Q_{G_{n} \cap E} V$ approaches zero as $n$ tends to infinity as in the proof of (2.8). Thus, letting $n$ approach infinity, we obtain $\gamma \bar{V} \geq \mu \bar{V}$, or $\eta \geq \pi$.

(ii) Suppose $\xi$ strongly dominates $\pi \in \operatorname{Exc}(U)$ so that $\xi=\pi+\nu$ for some $\nu \in \operatorname{Exc}(U)$. As in (i), there are measures $\mu$ and $\lambda$ on $F$ so that $q \pi=\mu \bar{V}$ and $q \nu=\lambda \bar{V}$. If we assume, in addition, that $\pi$ strongly dominates no nonzero measure in $\operatorname{Pot}(U)$, then $\mu$ is carried by $F-E$. In this case, we have

$$
(h \rho) V+\gamma \bar{V}=\mu \bar{V}+\left(1_{E} \lambda\right) \bar{V}+\left(1_{F-E} \lambda\right) \bar{V} .
$$

Set $\delta=(h \rho)+1_{E} \lambda$, and choose a decreasing sequence $\left(G_{n}\right)$ of open sets in $\bar{E}$ containing $\bar{E}-E$ so that $T_{G_{n}}$ increases to $T_{\bar{E}-E}, \bar{Q}^{\delta}$ a.s. As in (i), we have

$$
(h \rho) \bar{Q}_{G_{n}} \bar{V}+\gamma \bar{Q}_{G_{n}} \bar{V}=\mu \bar{Q}_{G_{n}} \bar{V}+\left(1_{E} \lambda\right) \bar{Q}_{G_{n}} \bar{V}+\left(1_{F-E} \lambda\right) \bar{Q}_{G_{n}} \bar{V} .
$$

Since $\gamma \bar{Q}_{G_{n}}=\gamma, \mu \bar{Q}_{G_{n}}=\mu,\left(1_{F-E} \lambda\right) \bar{Q}_{G_{n}}=1_{F-E} \lambda$ and $\lim _{n}(h \rho) \bar{Q}_{G_{n}} \bar{V}=$ $\lim _{n}\left(1_{E} \lambda\right) \bar{Q}_{G_{n}} \bar{V}=0$, we obtain upon letting $n$ increase to infinity that $\gamma \bar{V}=$ $\mu \bar{V}+\left(1_{F-E} \lambda\right) \bar{V}$. That is, $\eta$ strongly dominates $\pi$.

(iii) Suppose $\xi$ strongly dominates $\nu U$. Then $\xi=\nu U+\mu$ for some $\mu \in \operatorname{Exc}(U)$. Choose $\mu_{n}$ on $E$ with $\mu_{n} U$ increasing to $\mu$. Then $\left(\nu+\mu_{n}\right) U$ increases to $\xi$. From the proof of $(2.2)$, there is a subsequence $\mu_{n_{k}}$ so that $h\left(\nu+\mu_{n_{k}}\right)$ converges weakly to a measure $\bar{\lambda}$ on $\bar{E}$, where $h=U q$. Since $h \nu \leq \bar{\lambda}, h \nu=(h \nu) \bar{Q}_{0} \leq \bar{\lambda} \bar{Q}_{0}=\lambda$, and so $h \nu \leq 1_{E} \lambda$. Hence, $\nu \leq h^{-1} 1_{E} \lambda=\rho$, so $\rho-\nu$ is a positive measure. Therefore, $\nu U$ is strongly dominated by $\rho U$. Q.E.D. 
(2.11) REMARK. It is immediate from (2.10) that given $\xi \in \operatorname{Exc}(U)$, then $\xi \in \operatorname{Har}(U)$ if and only if $\xi$ strongly dominates no nonzero measure in $\operatorname{Pot}(U)$, and that $\xi \in \operatorname{Pot}(U)$ if and only if $\xi$ strongly dominates no nonzero measure in $\operatorname{Har}(U)$.

3. Weak duality: Elementary results. Fix two Borel right processes $X=$ $\left(\Omega, \mathcal{F}, \mathcal{F}_{t}, X_{t}, \theta_{t}, P^{x}\right)$ and $\hat{X}=\left(\hat{\Omega}, \hat{\mathcal{F}} \hat{\mathcal{F}}_{t}, \hat{X}_{t}, \hat{\theta}_{t}, \hat{P}^{x}\right)$ on a Lusin topological space $(E, \mathcal{E})$. In this section, and for the rest of the paper, we assume that $X$ and $\hat{X}$ are in weak duality with respect to a fixed $\sigma$-finite measure $m$ on $E$; that is, conditions (1.1) and (1.2) are assumed to hold. If $\mu$ is any measure on $(E, \mathcal{E})$, let $(f, g)_{\mu}=\int f g d \mu$. We shall write $(f, g)$ for $(f, g)_{m}$. Thus (1.1) may be rewritten as

$$
\left(P_{t} f, g\right)=\left(f, \hat{P}_{t} g\right)
$$

for $f, g \in \mathcal{E}^{+}$. It is an immediate consequence of (3.1) that $m$ is both excessive and coexcessive. Also

$$
\left(U^{\alpha} f, g\right)=\left(f, \hat{U}^{\alpha} g\right)
$$

As stated in the Introduction we shall suppose that $X$ and $\hat{X}$ are transient throughout this paper. That is, there is a function $q \in \mathcal{E}$ with $0<q \leq 1$ such that $U q \leq 1$ and $\hat{U} q \leq 1$. Of course, our results have analogs for $\alpha>0$ which are valid without this assumption of transience.

(3.3) Definition. $S^{\alpha}=S^{\alpha}(U)$ (resp. $\hat{S}^{\alpha}=S^{\alpha}(\hat{U})$ ) is the collection of Borel measurable functions on $E$ which are $\alpha$-excessive for $X$ (resp. $\hat{X}$ ) and which are finite a.e. $m$.

If $f$ is a universally measurable $\alpha$-excessive function, then there is a Borel measurable $\alpha$-excessive function $g$ with $f=g$ except on a finely open $m$-polar set [5, (6.11)].

(3.4) Definition. $\operatorname{Exc}^{\alpha}(U)$ (resp. $\left.\operatorname{Exc}^{\alpha}(\hat{U})\right)$ is the collection of $\sigma$-finite $\alpha$-excessive measures for $X$ (resp. $\hat{X}$ ).

As usual, $S^{0}, \hat{S}^{0}, \operatorname{Exc}^{0}(U)$ and $\operatorname{Exc}^{0}(\hat{U})$ will be written $S, \hat{S}, \operatorname{Exc}(U)$ and $\operatorname{Exc}(\hat{U})$.

(3.5) Definition. A function $u \in S^{\alpha}$ will be called the $\alpha$-potential of a measure $\mu$ if $u m=\mu \hat{U}^{\alpha}$. We also say $\mu$ has $\alpha$-potential $u$ in this case, and we use the notation $u=U^{\alpha}(\mu)$.

IMPORTANT REMARK. By the uniqueness theorem $[4,(1.1)]$ if $\mu \hat{U}^{\alpha}$ and $\nu \hat{U}^{\alpha}$ are $\sigma$-finite and equal, then $\mu=\nu$. Consequently $u$ determines $\mu$ uniquely in (3.5). Conversely $\mu$ determines $U^{\alpha}(\mu)$ a.e. $m$.

The terminology and notation above is motivated by that used in strong duality (see Chapter VI of [1]). There, $\mu \hat{U}^{\alpha}(d x)=U^{\alpha} \mu(x) m(d x)$, and $U^{\alpha} \mu(x)$, the $\alpha$ potential of $\mu$, is determined everywhere (not just $m$ a.e.); see (1.5).

(3.6) Proposition. If $f \in S^{\alpha}$, then $f m \in \operatorname{Exc}^{\alpha}(\hat{U})$. If $\hat{\mu} \in \operatorname{Exc}^{\alpha}(\hat{U})$ is absolutely continuous with respect to $m$, then there is an $f \in S^{\alpha}$ with $\hat{\mu}=f m$.

Proof. Let $f \in S^{\alpha}$, and set $\hat{\mu}=f m$. Then

$$
\hat{\mu} \hat{P}_{t}^{\alpha}(g)=\left(f, \hat{P}_{t}^{\alpha} g\right)=\left(P_{t}^{\alpha} f, g\right) \leq(f, g)=\hat{\mu}(g)
$$

for all $g \in \mathcal{E}^{+}$. Since $f<\infty$ a.e. $m$., $\hat{\mu}$ is $\sigma$-finite, and so $\hat{\mu} \in \operatorname{Exc}^{\alpha}(\hat{U})$. 
Now suppose that $\hat{\mu} \in \operatorname{Exc}^{\alpha}(\hat{U})$ and $\hat{\mu} \ll m$. Then $\hat{\mu}=h m$ for some function $h \in \mathcal{E}^{+} ; h<\infty m$ a.e. since $\hat{\mu}$ is $\sigma$-finite. For every $g \in \mathcal{E}^{+}$,

$$
\left(P_{t}^{\alpha} h, g\right)=\left(h, \hat{P}_{t}^{\alpha} g\right)=\hat{\mu} \hat{P}_{t}^{\alpha} g
$$

increases to $\hat{\mu}(g)=(h, g)$ as $t$ decreases to zero. Hence, $h$ is $m$ - $\alpha$-excessive [5, (6.14)]. By $[\mathbf{5},(6.19)]$, there is a Borel measurable $\alpha$-excessive $f$ with $f=h$ a.e. $m$ so that $f \in S^{\alpha}$ and $\hat{\mu}=f m$. Q.E.D.

Now we single out the class of measures $\mu$ for which $U^{\alpha}(\mu)$ can be defined. This class will play an important role in subsequent sections.

(3.7) Definition. Let $\mathcal{M}^{\alpha}$ (resp. $\hat{\mathcal{M}}^{\alpha}$ ) be the class of $\sigma$-finite measures $\mu$ on $E$ not charging cofinely open $m$-copolar sets and with $\mu \hat{U}^{\alpha} \sigma$-finite (resp. finely open $m$-polar sets and with $\mu U^{\alpha} \sigma$-finite).

Note that $m$ belongs to both $\mathcal{M}^{\alpha}$ and $\hat{\mathcal{M}}^{\alpha}$ if $\alpha>0$, but may not belong to $\mathcal{M}=\mathcal{M}^{0}$ or $\hat{\mathcal{M}}=\hat{\mathcal{M}}^{0}$. This occurs, for example, if $X$ and $\hat{X}$ are Brownian motion in $\mathbf{R}^{3}$ and $m$ is Lebesgue measure on $\mathbf{R}^{3}$.

(3.8) Proposition. Let $\mu$ be a measure on $E$. The following statements are equivalent.

(i) $\mu \in \mathcal{M}^{\alpha}$.

(ii) $\mu \hat{U}^{\alpha}$ is $\sigma$-finite and $\mu \hat{U}^{\alpha} \ll m$.

(iii) $\mu$ has an $\alpha$-potential $U^{\alpha}(\mu) \in \mathcal{S}^{\alpha}$.

(iv) $\mu \hat{U}^{\alpha}$ is $\sigma$-finite and $m$-copolar sets are $\mu$-copolar.

ProOF. (i) $\Rightarrow$ (ii). We need only show that if $A \in \mathcal{E}$ and $m(A)=0$, then $\mu \hat{U}^{\alpha}(A)=0$. Define a cofinely open Borel set by setting $B=\left\{x: \hat{U}^{\alpha}(x, A)>0\right\}$. If $\beta>\alpha, m \hat{U}^{\beta}(A) \leq(1 / \beta) m(A)=0$. By the monotone convergence theorem, $0=\lim _{\beta \downarrow \alpha} m \hat{U}^{\beta}(A)=m \hat{U}^{\alpha}(A)$. Therefore, $m(B)=0$. Since cofinely open $m$-null sets are $m$-copolar, $B$ is $m$-copolar. Thus $\mu(B)=0$ (since $\mu \in \mathcal{M}^{\alpha}$ ) and we conclude that $\mu \hat{U}^{\alpha}(A)=0$.

(ii) $\Rightarrow\left(\right.$ iii). Since $\mu \hat{U}^{\alpha} \in \operatorname{Exc}^{\alpha}(\hat{U})$, there is a function $U^{\alpha}(\mu) \in S^{\alpha}$ with $\mu \hat{U}^{\alpha}=$ $U^{\alpha}(\mu) \cdot m$ by $(3.6)$.

(iii) $\Rightarrow(\mathrm{i})$. Since $\mu \hat{U}^{\alpha}=U^{\alpha}(\mu) \cdot m$ and $U^{\alpha}(\mu) \in S^{\alpha}, \mu \hat{U}^{\alpha}$ is $\sigma$-finite and $\mu \hat{U}^{\alpha}$ does not charge cofinely open $m$-null sets. Therefore, neither does $\mu$. Since cofinely open $m$-copolar sets are $m$-null, $\mu \in \mathcal{M}^{\alpha}$.

(i) $\Leftrightarrow$ (iv). A measure $\mu$ satisfying (iv) clearly in $\mathcal{M}^{\alpha}$ since cofinely open $\mu$-copolar sets are $\mu$-null sets. Conversely, if $\mu \in \mathcal{M}^{\alpha}$, let $A$ be a Borel $m$-copolar set. Then $B=\left\{x: \hat{P}^{x}\left(T_{A}<\infty\right)>0\right\}$ is cofinely open and $m(B)=0$. Thus $\mu(B)=0$ so $\hat{P}^{\mu}\left(T_{A}<\infty\right)=0$. Q.E.D.

In the case of standard processes in strong duality, $m$-polar $=$ polar $=$ copolar $=m$ copolar, and the empty set is the only cofinely (or finely) open polar set.

Let $\mu \in \mathcal{M}$. Then $\mu$ is necessarily $\sigma$-finite and $\mu$ does not charge cofinely open $m$-copolar sets. Getoor and Sharpe $[\mathbf{5},(11.11)]$ showed that a $\sigma$-finite measure $\mu$ is the Revuz measure of a natural homogeneous random measure if and only if $\mu$ does not charge any $m$-copolar set. We shall not use this result, so we refer the reader to [5] for details.

(3.9) Proposition. Let $\mu \in \mathcal{M}^{\alpha}$ and let $u=U^{\alpha}(\mu)$. If $v \in S^{\alpha}$ and $v \leq u$ a.e. $m$, then $v=U^{\alpha}(\nu)$ for some measure $\nu \in \mathcal{M}^{\alpha}$. 
Proof. $\xi=v m \in \operatorname{Exc}^{\alpha}(\hat{U})$ by (3.6). But $\xi \leq u m=\mu \hat{U}^{\alpha}$, and hence by $[4$, (4.2)], $\xi=\nu \hat{U}^{\alpha}$ for some measure $\nu$ on $E$. Then $\nu \hat{U}^{\alpha}=v m$. Thus $\nu \in \mathcal{M}^{\alpha}$ and $v=U^{\alpha}(\nu)$. Q.E.D.

The following result is simple and interesting, and will be used in $\S 4$ to translate the results of $\S 2$ from excessive measures to excessive functions. If $B \in \mathcal{E}$, let

$$
\begin{gathered}
T_{B}=\inf \left\{t>0: X_{t} \in B\right\}, \quad S_{B}=\inf \left\{t: 0<t<\varsigma, X_{t-} \in B\right\}, \\
P_{B}^{\alpha} f(x)=E^{x}\left[e^{-\alpha T_{B}} f\left(X_{T_{B}}\right)\right], \quad P_{B-}^{\alpha} f(x)=E^{x}\left[e^{-\alpha S_{B}} f\left(X_{S_{B}}\right)\right],
\end{gathered}
$$

and $\hat{T}_{B}, \hat{S}_{B}, \hat{P}_{B}^{\alpha}$ and $\hat{P}_{B-}^{\alpha}$ are the corresponding objects for $\hat{X}$.

(3.10) Proposition. Let $\mu \in \mathcal{M}^{\alpha}$, and let $B \in \mathcal{E}$. Then $\mu \hat{P}_{B}^{\alpha}$ and $\mu \hat{P}_{B-}^{\alpha}$ are in $\mathcal{M}^{\alpha}$, and their $\alpha$-potential functions are $P_{B-}^{\alpha} U^{\alpha}(\mu)$ and $P_{B}^{\alpha} U^{\alpha}(\mu)$, respectively.

Proof. $\mu \hat{P}_{B}^{\alpha} \hat{U}^{\alpha} \leq \mu \hat{U}^{\alpha}$ and $\mu \hat{P}_{B-}^{\alpha} \hat{U}^{\alpha} \leq \mu \hat{U}^{\alpha}$, so by (3.8)(ii), $\mu \hat{P}_{B}^{\alpha}$ and $\mu \hat{P}_{B-}^{\alpha}$ are in $\mathcal{M}^{\alpha}$. The assertions about their $\alpha$-potential functions amount to $\mu \hat{P}_{B}^{\alpha} \hat{U}^{\alpha}=$ $P_{B-}^{\alpha} U^{\alpha}(\mu) \cdot m$ and $\mu \hat{P}_{B-}^{\alpha} \hat{U}^{\alpha}=P_{B}^{\alpha} U^{\alpha}(\mu) \cdot m$, respectively, and these are proved exactly as in $[5,(11.6)]$. Q.E.D.

4. The first two characterizations of potentials. In this section we "dualize" work in $\S 2$ to obtain results on excessive functions. The assumptions of $\S 3$ remain in force: $X$ and $\hat{X}$ are transient Borel right processes in weak duality with respect to $m$. Since we want to phrase our results in this section in terms of the excessive functions of $X$, we will need to use the results of $\S 2$ applied to the dual process $\hat{X}$. Thus, if $\xi \in \operatorname{Exc}(\hat{U})$ and $B \in \mathcal{E}, \hat{L}_{B} \xi=\lim _{n} \mu_{n} \hat{P}_{B} \hat{U}$, where $\left(\mu_{n}\right)$ is a sequence of measures on $E$ chosen so that $\mu_{n} \hat{U}$ increases to $\xi$. Also recall the operator $P_{B-}$ defined above (3.10).

(4.1) Proposition. If $\xi=u m$ with $u \in S$, then $\hat{L}_{B} \xi=\left(P_{B-} u\right) \cdot m$ for every $B \in \mathcal{E}$.

ProOF. Choose measures $\mu_{n}$ on $E$ so that $\mu_{n} \hat{U}$ increases to $\xi$. Then $\mu_{n} \hat{U} \ll m$, so $\mu_{n} \hat{U}=u_{n} m$ with $u_{n} \in S$. We may assume $u_{n} \leq u$ by replacing $u_{n}$ with $\min \left(u_{n}, u\right)$. It is clear that $u_{n} \leq u_{n+1}, m$ a.e. Hence $w \equiv \lim \inf u_{n}=u, m$ a.e., $P_{t} w \leq w$ and $w \leq u$. Since $m P_{t}(\{w \neq u\}) \leq m(\{w \neq u\})=0, P_{t} w=P_{t} u$ a.e. $m$. Thus if we let $v=\lim _{t \downarrow 0} P_{t} w$, we get that $v=u, m$ a.e., $v \in S$ and $v \leq w \leq u$. Set

$$
G=\bigcup_{n}\left\{u_{n}>u_{n+1}\right\} \cup\{v \neq u\} .
$$

Then $G$ is finely open and $m$-null. If $\eta \in \hat{\mathcal{M}}$ is equivalent to $m$, then $G$ is $\eta$-null. By (3.10), $\eta P_{B-} \in \hat{\mathcal{M}}$, so $G$ is $\eta P_{B-}$ polar (3.8), and hence $m P_{B-}$ polar. In particular $m P_{B-}$ does not charge $G$, so

$$
\hat{L}_{B} \xi=\lim _{n} \mu_{n} \hat{P}_{B} \hat{U}=\lim _{n}\left(P_{B-} u_{n}\right) \cdot m=\left(P_{B-} u\right) \cdot m,
$$

where the second equality is obtained from (3.10) and the third is obtained by applying the monotone convergence theorem since $u_{n}$ increases to $u$ off $G$. Q.E.D.

Now we characterize potentials in terms of balayage by "dualizing" (2.8). We first recall the "classical case" discussed in VI-2 of [1], where $X$ is a standard process in strong duality with a standard process $\hat{X}$ on an LCCB state space $E ;\left(\hat{U}^{\alpha}\right)_{\alpha \geq 0}$ 
is assumed to be strong Feller. Then any $u \in S$ is locally $m$-integrable and $u$ can be decomposed uniquely as $u=U \mu+h$, where $U \mu$ is the potential of a measure $\mu$ on $E$, and $h$ is an excessive function with the property that $P_{D} h=h$ whenever $D$ is the complement of a compact subset of $E$ [1, VI-2.11]. Moreover, a function $u \in S$ is the potential of a measure, $U \mu$, if and only if $\lim _{n} P_{K_{n}^{c}} u=0$ a.e. $m$, where $\left(K_{n}\right)$ is a fixed increasing sequence of compact subsets of $E$ such that $K_{n} \subset$ interior $\left(K_{n+1}\right)$ and $E=\bigcup K_{n}$.

Let us now return to discussing the case of weak duality. What is the correct necessary and sufficient condition characterizing potentials in terms of hitting operators in general? To answer this, we recall some notation of $\S 2$, but now for the process $\hat{X}$. Let $u \in S$ so that $\xi=u m \in \operatorname{Exc}(\hat{U})$ (3.6). Choose $q$ as in $\S 2$ so that $\xi(q)<\infty$ and $\hat{U} q \leq 1$. Set $\hat{V} g(x)=\hat{U} q(x)^{-1} \hat{U}(q g)(x)$, and compactify $E$ with the Ray cone generated by $\left(\hat{V}^{\alpha}\right)_{\alpha \geq 0}$ and $\left(\hat{W}^{\alpha}\right)_{\alpha>0}$ as in $\S 2$ to obtain a space $\hat{E}$. Recall that $\mathcal{K}(\hat{E})$ is the collection of subsets of $E$ which are compact in the topology of $\hat{E}$, and that $K(\hat{E}) \subset \mathcal{E}$. Also $K^{c}=E-K$ if $K \in \mathcal{K}(\hat{E})$.

(4.2) THEOREM. Let $u \in S$. Then $u$ is the potential of a measure in $\mathcal{M}, u=$ $U(\mu)$, if and only if $\inf \left\{P_{K^{c}-} u: K \in \mathcal{K}(\hat{E})\right\}=0$, a.e. $m$ (equivalently, um $\in$ $\operatorname{Pot}(\hat{U}))$.

Before starting the proof, we emphasize once again that $\hat{E}$ and $\mathcal{K}(\hat{E})$ may change with different choices of $q$. Of course, a similar result characterizing when a function $\hat{u} \in \hat{S}$ is the copotential of a measure $\hat{\mu} \in \hat{\mathcal{M}}$ holds as well.

Proof. Let $\xi=u m$. Then $\xi \in \operatorname{Pot}(\hat{U})$ if and only if there is a measure $\mu$ on $E$ with $\mu \hat{U}=u m$. That is, $u=U(\mu)$. If we use (4.1), then (2.8) translates into the following statement: $\xi \in \operatorname{Pot}(\hat{U})$ if and only if $\inf \left\{P_{K^{c}-} u \cdot m: K \in \mathcal{K}(\hat{E})\right\}=0$. That is, $u=U(\mu)$ if and only if $\inf \left\{P_{K^{c}-} u: K \in \mathcal{K}(\hat{E})\right\}=0$ a.e. $m$. Q.E.D.

In the "classical case" discussed before (4.2), where $X$ and $\hat{X}$ are standard processes in strong duality with $\left(\hat{U}^{\alpha}\right)$ a strong Feller resolvent on an LCCB state space, $q$ may be chosen so that $\hat{U} q$ is bounded and continuous on $E$ and $\mathcal{K}(\hat{E})$ is the collection of sets in $E$ which are compact in $E$. Moreover, $P_{B-}=P_{B}$ for standard processes, so (4.2) "almost" gives the well-known result in the classical case. (One needs to apply a standard argument permitting one to take the infimum over one fixed sequence of compact sets rather than over all compact sets. It is certainly necessary to allow the infimum over all sets in $\mathcal{K}(\hat{E})$ in the general case-one fixed sequence simply will not do.)

For the next definition recall the definition of $\operatorname{Har}(\hat{U})$ in $(2.5)$ and its characterization in (2.8). Of course, (2.5) and (2.8) must be applied to $\hat{X}$.

(4.3) DEFINITION. $\nLeftarrow \equiv\{u \in S: u m \in \operatorname{Har}(\hat{U})\}$.

The following characterization of $\nVdash$ is an immediate consequence of $(2.8)$ and (4.1).

(4.4) Proposition. $h \in \mathcal{H}$ if and only if $P_{K^{c}-} h=h$ a.e. $m$ for every $K \in$ $\mathcal{K}(\hat{E})$.

We come now to the first version of the Riesz decomposition for excessive functions. 
(4.5) TheOREM. Let $u \in S$. Then $u=U(\mu)+h$ a.e. $m$ where $\mu \in \mathcal{M}$ and $h \in \mathcal{H}$. Moreover, $\mu$ is unique and $h$ is unique a.e. $m$.

PROOF. Applying the duals of (2.2), (2.5) and (2.8) to $u m \in \operatorname{Exc}(\hat{U})$, we may write $u m=\mu \hat{U}+\eta$ where $\mu$ is a measure carried by $E$ and $\eta \in \operatorname{Har}(\hat{U})$. Clearly $\mu \hat{U}$ and $\eta$ are absolutely continuous with respect to $m$. By (3.8), $\mu \in \mathcal{M}$ and $\mu \hat{U}=U(\mu) \cdot m$, while (3.6) gives $\eta=h m$ with $h \in S$. Therefore $u=U(\mu)+h$ a.e. $m$, and $h \in \mathcal{H}$ since $\eta \in \operatorname{Har}(\hat{U})$. The uniqueness assertions follow from those of (2.2). Q.E.D.

Next we shall characterize $\not$ and potentials of measures in $\mathcal{M}$ in terms of the appropriate strong order for weak duality.

(4.6) DEFinition. $u \in S$ is said to strongly $m$-dominate $v \in S$ if there exists $w \in S$ with $u=v+w$ a.e. $m$. If the equality holds everywhere we say that $u$ strongly dominates $v$.

(4.7) LEMMA. Let $u \in S$. Then $u \in \nVdash$ if and only if $u$ strongly m-dominates the potential of no nonzero measure in $\mathcal{M}$.

Proof. $u \in \nVdash$ if and only if $u m \in \operatorname{Har}(\hat{U})$. By (2.10)(ii) this means $u m$ strongly dominates no nonzero measure in $\operatorname{Pot}(\hat{U})$ - that is to say of the form $U(\mu) \cdot m$ with $\mu \in \mathcal{M}$. But $U(\mu)=0$ a.e. $m$ if and only if $\mu=0$. Q.E.D.

In the next theorem " $w$ is larger than $v$ " means $w \geq v$ a.e. $m$.

(4.8) THEOREM. Let $u \in S$ and $u=U(\mu)+h$ a.e. $m$ be the decomposition of (4.5). Then:

(i) $h$ is the largest element of $\&$ with $h \leq u$ a.e. $m$.

(ii) $h$ is the largest element of $\forall$ in the strong $m$-domination order which is strongly $m$-dominated by $u$.

(iii) $U(\mu)$ is the largest potential of a measure in $\mathcal{M}$ in either the ordinary order (up to $m$-null sets) or the strong $m$-domination order which is strongly $m$ dominated by $u$.

PROOF. If $\xi=u m$ and $\eta=v m$ with $u, v \in S$, then $\xi \leq \eta$ if and only if $u \leq v$ a.e. $m$, and $\xi$ is strongly dominated by $\eta$ if and only if $u$ is strongly $m$-dominated by $v$. Bearing in mind (4.3) and the fact that $u m \in \operatorname{Pot}(\hat{U})$ if and only if $u=U(\mu)$ for $\mu \in \mathcal{M},(4.8)$ is now a direct translation of the dual of (2.10). Q.E.D.

If the processes $X$ and $\hat{X}$ are in strong duality with respect to $m$, then two excessive functions which agree a.e. $m$ are identical. Also if $u, v \in S$ and $u \geq v$ a.e. $m$, then $u \geq v$. This leads to obvious simplifications in the statement of (4.8).

5. Time reversal and $h$-transforms. In this section, we collect several facts about time reversal and $h$-transforms which will be needed later. Most of the facts are well known. Some of the $h$-transform results can be found in [10]. There is a complete exposition in the Paris lecture notes of J. B. Walsh [11].

Let $X=\left(\Omega, \mathcal{F}, \mathcal{F}_{t}, X_{t}, \theta_{t}, P^{x}\right)$ be a Borel right process canonically constructed on the space $\Omega$ of right continuous paths in $E \cup\{\Delta\}$. Let $P_{t}$ and $U^{\alpha}$ be the semigroup and resolvent of $X$, and let $h$ be a Borel measurable excessive function. Set $E_{h}=\{x: 0<h(x)<\infty\} \in \mathcal{E}$. As usual, a conditioned semigroup may be defined on $E_{h}$ by setting

$$
P_{t}^{h} f(x)=h(x)^{-1} P_{t}(f h)(x) \quad \text { for } x \in E_{h} .
$$


In a perhaps unusual move, we extend $P_{t}^{h}$ to be a semigroup on all of $E$ by setting

$$
P_{t}^{h} f(x)=f(x) \text { for } x \in E-E_{h} .
$$

It is well known that there exist probabilities $P^{x / h}$ for $x$ in $E_{h}$ so that $X^{h}=$ $\left(X_{t}, P^{x / h}\right)$ is a Borel right process with state space $E_{h}$ and semigroup $P_{t}^{h}$ restricted to $E_{h}$. Let $\omega_{x}$ be the path in $\Omega$ so that $\omega_{x}(t)=x$ for all $t \geq 0$. If $x \in E-E_{h}$, let $P^{x / h}$ be unit mass at $\omega_{x}$. Then $\left(X_{t}, P^{x / h}\right)_{x \in E}$ is a Borel right process with state space $E$ and semigroup $P_{t}^{h}$ for which $E_{h}$ and $E-E_{h}$ are absorbing.

Recall that $\mathcal{F}^{0}=\sigma\left\{X_{s}: s \geq 0\right\}$ and $\mathcal{F}_{t}^{0}=\sigma\left\{X_{s}: s \leq t\right\}$. If $Q$ is any probability on $\left(\Omega, \mathcal{F}^{0}\right), \mathcal{F}^{Q}$ is the $Q$-completion of $\mathcal{F}^{0}, \mathcal{N}^{Q}$ is the ideal of $Q$-null sets in $\mathcal{F}^{Q}$, and $\mathcal{F}_{t}^{Q}=\sigma\left(\mathcal{F}_{t}^{0}, \mathcal{N}^{Q}\right)$. Then $\mathcal{F}_{t}^{*}=\bigcap_{Q} \mathcal{F}_{t}^{Q}, \mathcal{F}_{t+}^{*}=\bigcap_{s>t} \mathcal{F}_{s}^{*}$.

Let $S$ be an arbitrary $\left(\mathcal{F}_{t}\right)$ stopping time. One may always choose an $\left(\mathcal{F}_{t+}^{*}\right)$ stopping time $T$ so that $S=T, P^{\mu}$ a.s. for every measure $\mu$ on $E$ [8]. Thus, we may restrict our attention to $\left(\mathcal{F}_{t+}^{*}\right)$ stopping times in the next well-known result, a proof of which may be found in $[\mathbf{1 1}]$.

(5.1) PROPOSITION. If $T$ is an $\left(\mathcal{F}_{t+}^{*}\right)$ stopping time and $F \in b \mathcal{F}_{T+}^{*}$, then

$$
E^{x / h}[F ; T<\varsigma]=h(x)^{-1} E^{x}\left[F h\left(X_{T}\right)\right]
$$

for every $x$ in $E_{h}$.

(5.2) COROLlaRY. If $X$ has left limits a.s. on $] 0, \varsigma\left[\right.$, then so does $X^{h}$.

In the case that $X$ has left limits a.s. on $] 0, \varsigma$, we may use the canonical space $\Omega$ of paths $\omega:[0, \infty[\rightarrow E \cup\{\Delta\}$ which are right continuous on $[0, \infty[$ with left limits in $E$ on $] 0, \varsigma[$.

Let $(I, I, \nu)$ be a $\sigma$-finite measure space, and let $\left\{u_{z}(x): z \in I\right\}$ be a family of functions indexed by $I$ so that each $u_{z}$ is excessive for $X$. Suppose that $(z, x) \mapsto$ $u_{z}(x)$ is $I \times \mathcal{E}$ measurable. Set $h(x)=\int u_{z}(x) \nu(d z)$ so that $h \in \mathcal{E}$ is excessive. For each $x \in E$ and for each $z \in I$, let $P_{z}^{x}=P^{x / u_{z}}$.

(5.3) Proposition. For each $x \in E_{h}$ and for each $F \in \mathcal{F}^{*+}$,

$$
E^{x / h}[F]=h(x)^{-1} \int u_{z}(x) E_{z}^{x}[F] \nu(d z) .
$$

The next result shows that with the slightly unusual $h$-transform we have defined above, weak duality is preserved. Let $X=\left(\Omega, \mathcal{F}, \mathcal{F}_{t}, X_{t}, \theta_{t}, P^{x}\right)$ and $\hat{X}=$ $\left(\Omega, \hat{\mathcal{F}}, \hat{\mathcal{F}}_{t}, \hat{X}_{t}, \hat{\theta}_{t}, \hat{P}^{x}\right)$ be Borel right processes in weak duality with respect to $m$ as in $\S 1$. We assume they are defined on the canonical space $\Omega$ mentioned after Corollary (5.2) so the results above apply.

(5.4) Proposition. If $h \in S$, then $X^{h}$ and $\hat{X}$ are Borel right processes in weak duality on $E$ with respect to $h m$.

Proof. Recall that $m(h=\infty)=0$ and $h \in \mathcal{E}$ by definition of $S$. Therefore, $P_{t}^{h} f \in \mathcal{E}^{+}$whenever $f \in \mathcal{E}^{+}$, so $X^{h}$ is a Borel right process. Since $h<\infty$ a.e. $m$, the measure $h m$ is carried by $E_{h}$. Now $P_{t} h(x)=0$ if $h(x)=0$. Thus if $f$ and $g$ are bounded positive Borel functions on $E$, then

$$
\left(f, P_{t}^{h} g\right)_{h m}=\int_{E_{h}} f\left(P_{t}^{h} g\right) h d m=\int_{E_{h}} f P_{t}(h g) d m=\left(f, P_{t}(h g)\right)
$$


since $m(h=\infty)=0$ and $P_{t}(h g)=0$ on $\{h=0\}$. But

$$
\left(f, P_{t}(h g)\right)=\left(\hat{P}_{t} f, h g\right)=\left(\hat{P}_{t} f, g\right)_{h m},
$$

which gives the desired result. Q.E.D.

Finally, we need Nagasawa's theorem on time reversal. A proof of this is given in $\S 14$ of [5] for a restricted class of initial measures for processes in weak duality. Since a "slightly unusual" definition of $P_{t}^{h}$ is being used here, one needs to check that Nagasawa's theorem holds if this version of $P_{t}^{h}$ is used. The modifications in the proof in [5] needed to extend it here are elementary, so we omit them and state the following result for the record. A cooptional time $L$ is an $F^{*}$-measurable random variable with $L \leq \varsigma$ and $L \circ \theta_{t}=(L-t)^{+}$for every $\omega$ in $\Omega$.

(5.5) THEOREM. Let $\mu \in \hat{\mathcal{M}}$, and let $\hat{u}=\hat{U}(\mu)$ be the copotential of $\mu$. If $L$ is a cooptional time for $X$, define

$$
\tilde{X}_{t}= \begin{cases}X_{(L-t)-} & \text { if } 0<t<L<\infty, \\ \Delta & \text { otherwise. }\end{cases}
$$

Under $P^{\mu},\left(\tilde{X}_{t}\right)_{t>0}$ is a homogeneous Markov process with semigroup $\left(\hat{P}_{t}^{\hat{u}}\right)$.

Note that (5.5) says nothing about determining the entrance law of $\tilde{X}_{t}$; it only gives information about the semigroup.

6. A time reversal theorem. We begin this section with two results about $\alpha$-potentials of measures. As in earlier sections, $X$ and $\hat{X}$ are transient Borel right processes in weak duality with respect to $m$. In particular (1.2) is being assumed.

Observe that $\mathcal{M}^{\alpha} \subset \mathcal{M}^{\beta}$ if $\alpha<\beta$. then

(6.1) Proposition. Let $\mu \in \mathcal{M}$ and set $u^{\alpha}=U^{\alpha}(\mu)$ for $\alpha \geq 0$. If $0 \leq \alpha<\beta$,

$$
u^{\alpha}=u^{\beta}+(\beta-\alpha) U^{\alpha} u^{\beta}=u^{\beta}+(\beta-\alpha) U^{\beta} u^{\alpha}
$$

except possibly on a finely open m-polar set.

PROOF. According to the resolvent equation,

$$
\mu \hat{U}^{\alpha}=\mu \hat{U}^{\beta}+(\beta-\alpha) \mu \hat{U}^{\beta} \hat{U}^{\alpha}=\mu \hat{U}^{\beta}+(\beta-\alpha) \mu \hat{U}^{\alpha} \hat{U}^{\beta} .
$$

If $g \in \mathcal{E}^{+}$, then $\mu \hat{U}^{\beta} \hat{U}^{\alpha}(g)=\left(u^{\beta}, \hat{U}^{\alpha} g\right)=\left(U^{\alpha} u^{\beta}, g\right)$ and similarly $\mu \hat{U}^{\alpha} \hat{U}^{\beta}(g)=$ $\left(U^{\beta} u^{\alpha}, g\right)$. Therefore $(6.2)$ holds a.e. $m$. Since each term in (6.2) is finely continuous, one obtains (6.1). Q.E.D.

Under strong duality the next result is a trivial consequence of Fubini's theorem.

(6.3) Proposition. Let $\mu \in \mathcal{M}$ and $\hat{\mu} \in \hat{\mathcal{M}}$. Then for each $\alpha \geq 0, \mu\left[\hat{U}^{\alpha}(\hat{\mu})\right]=$ $\hat{\mu}\left[U^{\alpha}(\mu)\right]$.

ProOF. Let $u^{\alpha}=U^{\alpha}(\mu)$ and $\hat{u}^{\alpha}=\hat{U}^{\alpha}(\hat{\mu})$. Since $\hat{u}^{\alpha} \in \hat{S}^{\alpha}, n \hat{U}^{\alpha+n} \hat{u}^{\alpha}$ increases to $\hat{u}^{\alpha}$ as $n$ increases to infinity. Therefore

$$
\mu\left(\hat{u}^{\alpha}\right)=\lim _{n} n \mu\left(\hat{U}^{\alpha+n} \hat{u}^{\alpha}\right)=\lim _{n} n\left(u^{\alpha+n}, \hat{u}^{\alpha}\right)=\lim _{n} n \hat{\mu}\left(U^{\alpha} u^{\alpha+n}\right) .
$$

From (6.1), $u^{\alpha}=u^{\alpha+n}+n U^{\alpha} u^{\alpha+n}$ except on a finely open $m$-polar set. But $\hat{\mu} \in \hat{M}$, and so this equality holds a.e. $\hat{\mu}$. Hence $n U^{\alpha} u^{\alpha+n} \leq u^{\alpha}$ a.e. $\hat{\mu}$ and consequently $\mu\left(\hat{u}^{\alpha}\right) \leq \hat{\mu}\left(u^{\alpha}\right)$. Similarly $\hat{\mu}\left(u^{\alpha}\right) \leq \mu\left(\hat{u}^{\alpha}\right)$. Q.E.D. 
Next comes the main result of this section. It is of obvious probabilistic interest and will also be used in $\S 7$ to obtain results about the potential theory of $X$ and $\hat{X}$. As in $\S 5$ we shall suppose without loss of generality in the remainder of this section that $\Omega=\hat{\Omega}$ is the canonical space of all right continuous maps $\omega$ from $\mathbf{R}^{+}$to $E \cup\{\Delta\}$ which have left limits in $E$ on $] 0, \varsigma(\omega)\left[\right.$. Then $X_{t}(\omega)=\hat{X}_{t}(\omega)=\omega(t)$ and the various processes are described by families of measures on $\Omega$. Thus a $u$-transform $X^{u}$ of $X$ is described by the family of measures $P^{x / u}$ and $X_{t}^{u}(\omega)=X_{t}(\omega)=\omega(t)$ is the coordinate map for each $t \geq 0$.

We define the reversal $\tilde{X}$ of $X$ as follows:

$$
\tilde{X}_{t}(\omega)= \begin{cases}X_{(\varsigma-t)-}(\omega) & \text { if } 0<t<\varsigma(\omega)<\infty \\ \Delta & \text { otherwise. }\end{cases}
$$

Note that $t \rightarrow \tilde{X}_{t}(\omega)$ is right continuous on $] 0, \infty[$ and has left limits in $E$ on $[0, \varsigma(\omega)]$ if $\varsigma(\omega)<\infty$. But $\tilde{X}_{0+}$ need not exist in general. Thus the map $r$ which sends $\omega$ into the trajectory $r \omega(t)=\tilde{X}_{t}(\omega)$ does not map $\Omega$ into $\Omega$.

(6.5) TheOREM. Let $\mu \in \mathcal{M}$ and $u=U(\mu)$. Let $\hat{\mu} \in \hat{\mathcal{M}}$ with $\hat{\mu}(u)<\infty$ and let $\hat{u}=\hat{U}(\hat{\mu})$. Then $\left(\tilde{X}_{t}, P^{u \hat{\mu} / u}\right)_{t>0}$ has the same law as $\left(X_{t}, \hat{P}^{\hat{u} \mu / \hat{u}}\right)_{t>0}$.

REMARKS. By (6.3), $\mu(\hat{u})=\hat{\mu}(u)<\infty$. Hence, $\mu(\hat{u}=\infty)=0=\hat{\mu}(u=\infty)$. Thus $u \hat{\mu}$ is carried by $E_{u}$ and $\hat{u} \mu$ is carried by $E_{\hat{\mu}}$. Hence $P^{u \hat{\mu} / u}$ and $\hat{P}^{\hat{u} \mu / \hat{u}}$ do not depend on our convention for extending $h$-transforms from $E_{h}$ to $E$ in $\S 5$. Of course, $u$ (resp. $\hat{u}$ ) is only determined up to a finely open $m$-polar (resp. cofinely open $m$-copolar) set, but we claim that $P^{u \hat{\mu} / u}$ (resp. $\left.\hat{P}^{\hat{u} \mu / \hat{u}}\right)$ is uniquely determined. To this end suppose $v \in S$ and $u=v$ a.e. $m$. Then $N=\{u \neq v\}$ is a finely open $m$-polar set. Since $\hat{\mu} \in \hat{\mathcal{M}}, \hat{\mu}(N)=0$ and so $u \hat{\mu}=v \hat{\mu}$ (as measures) and each is carried by $E_{u} \cap E_{v}$. Let $A=\left\{x: P^{x}\left(T_{N}<\infty\right)>0\right\}$. Then $A$ is a finely open $m$-polar set and hence $\hat{\mu}(A)=0$. If $x \in H \equiv A^{c} \cap N^{c} \cap E_{u} \cap E_{v}$ and $F \in \mathcal{F}_{t}^{0}, t>0$, then

$$
u(x) P^{x / u}[F, t<\varsigma]=E^{x}\left[F u\left(X_{t}\right)\right]=E^{x}\left[F v\left(X_{t}\right)\right]=v(x) P^{x / v}[F, t<\varsigma] .
$$

Hence $P^{x / u}[F, t<\varsigma]=P^{x / v}[F, t<\varsigma]$ for $F \in F_{t}^{0}$ and $t>0$. But $F \cap\{t<$ $\varsigma\}, F \in \mathcal{F}_{t}^{0}$, is a $\pi$-system generating $\mathcal{F}^{0}$, and since $u \hat{\mu}=v \hat{\mu}$ is carried by $H$ above, $P^{u \hat{\mu} / u}=P^{v \hat{\mu} / v}$. Similarly $\hat{P}^{\hat{u} \mu / \hat{u}}$ does not depend on the particular chace of $\hat{u}$.

ProOF. By (5.4), $X^{u}$ and $\hat{X}$ are in weak duality with respect to $u m$. According to Nagasawa's theorem (5.5) applied to $\left(X^{u}, \hat{X}, u m\right),\left(\tilde{X}_{t}\right)_{t>0}$ under $P^{u \hat{\mu} / u}$ is a right continuous homogeneous Markov process whose transition semigroup we may compute as follows: Let $\left(U_{u}^{\alpha}\right)$ be the resolvent of $X^{u}$. If $g \in b \mathcal{E}^{+}$, then

$$
(u \hat{\mu}) U_{u}(g)=\int_{E_{u}} \hat{\mu}(d x) u(x) \frac{1}{u(x)} U(g u)(x)
$$

since $u \hat{\mu}$ is carried by $E_{u}$. But $\alpha U^{\alpha} u \leq u$, and it follows that if $u(x)=0$, then $U^{\alpha} u(x)=0$ for $\alpha>0$. Letting $\alpha$ decrease to 0 gives $U u(x)=0$, and so $U(g u)$ vanishes on $\{u=0\}$. Thus the right side of $(6.6)$ may be written $\hat{\mu} U(g u)=(\hat{u}, g u)=$ $u m(g \hat{u})$. Hence $\hat{u}$ is the copotential of the measure $u \hat{\mu}$ relative to $\left(X^{u}, \hat{X}, u m\right)$. Consequently by $(5.5)$ the semigroup of $\left(\tilde{X}_{t}\right)_{t>0}$ under $P^{u \hat{\mu} / u}$ is $\hat{P}_{t}^{\hat{u}}$. By its very 
definition $\hat{X}^{\hat{u}}$ has this semigroup and so to complete the proof of (6.5) we must check that the two processes in question have the same entrance law.

For this it suffices to show, for $\alpha>0$ and $f$ a bounded continuous function, that

$$
\int_{0}^{\infty} e^{-\alpha t} E^{u \hat{\mu} / u}\left[f\left(X_{(\varsigma-t)-}\right) ; t<\varsigma\right] d t=\int_{0}^{\infty} e^{-\alpha t} \hat{E}^{\hat{u} \mu / \hat{u}}\left[f\left(X_{t}\right)\right] d t
$$

since $\hat{\mu}(u)<\infty$ and $\mu(\hat{u})<\infty$ imply that each side of $(6.7)$ is finite and that each of the expectations in (6.7) is right continuous in $t$, at least on $] 0, \infty[$. We prepare two lemmas for the proof of (6.7).

(6.8) LemmA. Let $u^{\alpha}=U^{\alpha}(\mu)$. Then for each $\alpha>0, u(x) E^{x / u}\left(e^{-\alpha \varsigma}\right)=$ $u^{\alpha}(x)$ a.e. $\hat{\mu}$ and the exceptional set is contained in $\{u=\infty\} \cup\left\{u \neq u^{\alpha}+\alpha U^{\alpha} u\right\}$.

PROOF. If $u(x)<\infty$ and $\alpha>0$,

$$
\begin{aligned}
u(x) E^{x / u}\left(e^{-\alpha \varsigma}\right) & =\alpha u(x) E^{x / u} \int_{\zeta}^{\infty} e^{-\alpha t} d t \\
& =u(x)\left[1-\alpha U_{u}^{\alpha} 1(x)\right]=u(x)-\alpha U^{\alpha} u(x) .
\end{aligned}
$$

By (6.1), $u=u^{\alpha}+\alpha U^{\alpha} u$ except possibly on a finely open $m$-polar set. But $\hat{\mu} \in \hat{\mathcal{M}}$ and so $u=u^{\alpha}+\alpha U^{\alpha} u$ a.e. $\hat{\mu}$. Since $\hat{\mu}(u=\infty)=0$, we obtain (6.8).

(6.9) LEMMA. The set $\{u=\infty\} \cup\left\{u \neq u^{\alpha}+\alpha U^{\alpha} u\right\}$ in Lemma (6.8) is $\hat{\mu}$-polar $\left(\right.$ for $\left.\left(X_{t}, P^{x}\right)\right)$.

Proof. By (6.1), $\left\{u \neq u^{\alpha}+\alpha U^{\alpha} u\right\}$ is a finely open $m$-polar set, and hence $\hat{\mu}$-polar because of $\hat{\mu} \in \hat{\mathcal{M}}$ and the dual of (3.8). If $A \equiv\{u=\infty\}$, then $A$ is finely closed and $P_{A}(x, \cdot)$ is carried by $A$. Since $E^{\hat{\mu}}\left[u\left(X_{T_{A}}\right)\right] \leq \hat{\mu}(u)<\infty, P^{\hat{\mu}}\left(T_{A}<\infty\right)$ $=0$. Thus $A$ is $\hat{\mu}$-polar. Q.E.D.

We return now to the proof of (6.7). The left-hand side of (6.7) equals

$$
\begin{aligned}
E^{u \hat{\mu} / u} \int_{0}^{\varsigma} e^{-\alpha \varsigma \circ \theta_{t}} f\left(X_{t}\right) d t & =\int_{0}^{\infty} E^{u \hat{\mu} / u}\left[E^{X(t) / u}\left(e^{-\alpha \varsigma}\right) f\left(X_{t}\right) ; t<\varsigma\right] d t \\
& =\int_{0}^{\infty} d t \int u(x) \hat{\mu}(d x) E^{x / u}\left[E^{X(t) / u}\left(e^{-\alpha \varsigma}\right) f\left(X_{t}\right) ; t<\varsigma\right] .
\end{aligned}
$$

Applying (5.1) and remembering that $\hat{\mu}(u=\infty)=0$ this becomes

$$
\int_{0}^{\infty} d t \int \hat{\mu}(d x) E^{x}\left[u\left(X_{t}\right) E^{X(t) / u}\left(e^{-\alpha \varsigma}\right) f\left(X_{t}\right)\right]
$$

where we have used the fact that $E^{x}\left[u\left(X_{t}\right)\right] \leq u(x)$ so that the integral in $x$ above is really over $E_{u}$. In light of (6.8) and (6.9) we rewrite this as

$$
\int d t \int \hat{\mu}(d x) E^{x}\left[u^{\alpha}\left(X_{t}\right) f\left(X_{t}\right)\right]=\hat{\mu} U\left(u^{\alpha} f\right)=\left(\hat{u}, u^{\alpha} f\right) .
$$

Next, the right-hand side of (6.7) may be written $\int \hat{U}_{\hat{u}}^{\alpha} f(x) \hat{u}(x) \mu(d x)$. But $\mu(\hat{u}=\infty)=0$ and $\hat{U}^{\alpha}(f \hat{u})=0$ on $\{\hat{u}=0\}$, so this last expression becomes

$$
\int \mu(d x) \hat{U}^{\alpha}(f \hat{u})(x)=\mu \hat{U}^{\alpha}(f \hat{u})=\left(u^{\alpha}, f \hat{u}\right)=\left(\hat{u}, u^{\alpha} f\right) .
$$

Hence (6.7) holds and this completes the proof of (6.5). Q.E.D.

The following corollary, or more precisely its dual, will be used in $\S 7$. 
(6.10) COROLlaRY. Under the hypotheses of (6.5):

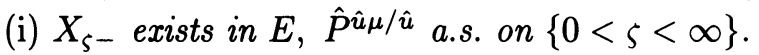

(ii) For each $f \in b \mathcal{E}, U^{\alpha} f\left(X_{\zeta}\right)_{-}$exists and equals $U^{\alpha} f\left(X_{\varsigma^{-}}\right), \hat{P}^{\hat{u} \mu / \hat{u}}$ a.s. on $\{0<\varsigma<\infty\}$.

Proof. Using the notation of (6.4), let $\tilde{\zeta}=\inf \left\{t>0: \tilde{X}_{t}=\Delta\right\}$. Then $\{0<\zeta<$ $\infty\}=\{0<\tilde{\zeta}<\infty\}$ and $\tilde{\zeta}=\zeta$ on this set. Now

$$
\begin{aligned}
& \hat{P}^{\hat{u} \mu / \hat{u}}\left[X_{\zeta-}\right. \text { exists in } E, U^{\alpha} f\left(X_{\zeta}\right)_{-} \text {exists and } \\
&\text { equals } \left.U^{\alpha} f\left(X_{\zeta^{-}}\right), 0<\zeta<\infty\right] \\
&= \hat{P}^{\hat{u} \mu / \hat{u}}\left[\tilde{X}_{0+} \text { exists in } E, U^{\alpha} f\left(\tilde{X}_{0}\right)_{+}\right. \text {exists and } \\
&\text { equals } \left.U^{\alpha} f\left(\tilde{X}_{0+}\right), 0<\tilde{\zeta}<\infty\right] \\
&= P^{u \hat{\mu} / u}\left[X_{0+} \text { exists in } E, U^{\alpha} f\left(X_{0}\right)_{+}\right. \text {exists and } \\
&\text { equals } \left.U^{\alpha} f\left(X_{0+}\right), 0<\zeta<\infty\right],
\end{aligned}
$$

where the second equality follows from the dual of (6.5). If $t>0$ and $x \in E_{u}$

$$
\begin{aligned}
& P^{x / u}\left[X_{0+} \text { exists in } E, U^{\alpha} f\left(X_{0}\right)_{+} \text {exists and equals } U^{\alpha} f\left(X_{0+}\right), t<\varsigma\right] \\
& \quad=u(x)^{-1} E^{x}\left[u\left(X_{t}\right) ; X_{0+} \text { exists in } E, U^{\alpha} f\left(X_{0}\right)_{+}\right. \text {exists and } \\
& \quad=u(x)^{-1} E^{x}\left[u\left(X_{t}\right) ; t<\varsigma\right],
\end{aligned}
$$

where the last equality follows from the very definition of a right process. Since $u$ is excessive this last term increases to one as $t$ decreases to zero. Combining this with the fact that $u \hat{\mu}$ is carried by $E_{u}$, the last expression in (6.11) is equal to

$$
P^{u \hat{\mu} / u}[0<\varsigma<\infty]
$$

which, using (6.5) once again, is equal to $\hat{P}^{\hat{u} \mu / \hat{u}}[0<\varsigma<\infty]$. Q.E.D.

Although it will not be needed in this paper, it is of interest to investigate to what extent the assumption $\hat{\mu}(u)<\infty$ may be weakened in (6.5). As example (6.13) shows it cannot be dropped altogether even if $\hat{\mu}$ is carried by $E_{u}$. However, we have the following positive result. The remarks following (6.5) apply equally here.

(6.12) Proposition. Let $\mu \in \mathcal{M}$ and $\hat{\mu} \in \hat{\mathcal{M}}$. Let $u=U(\mu)$ and $\hat{u}=\hat{U}(\hat{\mu})$. If $\mu(\hat{u}=\infty)=0$ and $\hat{\mu}(u=\infty)=0$, then the conclusion of $(6.5)$ is valid.

Proof. Since $\hat{\mu}$ is $\sigma$-finite and $\hat{\mu}(u=\infty)=0$ we may choose disjoint sets $E_{k} \in \mathcal{E}$ with $E=\bigcup E_{k}$ and $\int_{E_{k}} u d \hat{\mu}<\infty$ for each $k$. Let $\hat{\mu}_{k}=1_{E_{k}} \hat{\mu}$. Then $\hat{\mu}_{k} \in \hat{\mathcal{M}}, \hat{\mu}_{k}(u)<\infty$, and $\hat{\mu}=\sum \hat{\mu}_{k}$. Thus each $\hat{\mu}_{k}$ satisfies the hypotheses of (6.5). If $\hat{u}_{k}=\hat{U}\left(\hat{\mu}_{k}\right)$, then $\hat{u}=\sum \hat{u}_{k}$ a.e. $m$ because $\sum_{k} \hat{\mu}_{k} U=\hat{\mu} U$. Since $\hat{u}$ is only determined $m$ a.e. we may suppose $\hat{u}=\sum \hat{u}_{k}$. Now

$$
P^{u \hat{\mu} / u}=\int u(x) \hat{\mu}(d x) P^{x / u}=\sum P^{u \hat{\mu}_{k} / u} .
$$


On the other hand, using $(5.3)$ and $\mu(\hat{u}=\infty)=0$,

$$
\begin{aligned}
\hat{P}^{\hat{u} \mu / \hat{u}} & =\int \mu(d x) \hat{u}(x) \hat{P}^{x / \hat{u}}=\int_{E_{\hat{u}}} \mu(d x) \hat{u}(x) \hat{P}^{x / \hat{u}} \\
& =\int_{E_{\hat{u}}} \mu(d x) \sum \hat{u}_{k}(x) \hat{P}^{x / \hat{u}_{k}}=\sum \hat{P}^{\hat{u}_{k} \mu / \hat{u}_{k}},
\end{aligned}
$$

where the last equality follows because each $\hat{u}_{k}=0$ on $\{\hat{u}=0\}$. But (6.5) applies to each $\hat{\mu}_{k}$, and hence (6.12) is valid. Q.E.D.

We close this section with two examples, the first of which illustrates the delicacy of (6.12). The second is important for time reversal of processes in strong duality.

(6.13) EXAMPLE. The state space $E=\mathbf{R}, X$ is translation to the right at unit speed killed when it hits (singleton) $\{0\}$, and $\hat{X}$ translation to the left at unit speed killed when it hits $\{0\}$. Clearly $X$ and $\hat{X}$ are in strong duality relative to Lebesgue measure $m(d x)=d x$. Let $\mu=\varepsilon_{0}$. Then $\mu \hat{U}=1_{]-\infty, 0[} m$, so $u \equiv$ $U(\mu)=1_{]-\infty, 0[}$. Thus $\left.E_{u}=\right]-\infty, 0\left[\right.$. Define $\hat{\mu}(d x)=x^{-2} 1_{-\infty, 0[}(x) d x$. Then $\hat{u} \equiv \hat{U}(\hat{\mu})=|x|^{-1} 1_{]-\infty, 0]}(x)$. Now $u \hat{\mu}=\hat{\mu}$ while $X^{u}$ is simply the restriction of $X$ to ] $-\infty, 0\left[=E_{u}\right.$, and then extended to $E$ by our convention. On the other hand $\hat{u} \mu=\infty \cdot \varepsilon_{0}$ and the restriction of $\hat{u} \mu$ to $E_{\hat{u}}$ is zero! Thus there seems to be no transparent interpretation of $\hat{P}^{\hat{u} \mu / \hat{u}}$ in this case.

(6.14) EXAMPLE. Let $X$ and $\hat{X}$ be in strong duality relative to $m$. Let $u(x, y)$ be the potential density $(\alpha=0)$ in (1.4). Let $P^{x, y}=P^{x / u(\cdot, y)}$ and $\hat{P}^{y, x}=\hat{P}^{y / u(x, \cdot)}$. If $0<u(x, y)<\infty$, it is immediate from (6.5) with $\hat{\mu}=\varepsilon_{x}$ and $\mu=\varepsilon_{y}$ that $\left(\tilde{X}_{t}\right)_{t>0}$ under $P^{x, y}$ is equivalent to $\left(X_{t}\right)_{t>0}$ under $\hat{P}^{y, x}$. This is somewhat stronger than the known fact that $\hat{P}^{y, x}$ almost surely $\zeta<\infty$ and $X_{\zeta^{-}}=x$ (see [10 or 11]). It now follows that $\left(\tilde{X}_{t+}, P^{x, y}\right)_{t \geq 0}$ has the same law as $\left(X_{t}, \hat{P}^{y, x}\right)_{t \geq 0}$. Of course, the roles of $X$ and $\hat{X}$ may be interchanged in this discussion.

7. The third characterization of potentials. Again, in this section, $X$ and $\hat{X}$ are Borel right processes on $E$ in weak duality with respect to $m$. We also assume that $X$ and $\hat{X}$ are defined on the canonical path space $\Omega$ discussed following (5.2) and the proof of (6.3). We fix a countable dense set $\left(g_{n}\right)$ in $b \mathbf{C}_{u}^{+}(E)$ with $g_{1}=1$. Choose $q \in \mathcal{E}^{+}, 0<q \leq 1$, with $m(q)<\infty$ and $h \equiv \hat{U} q \leq 1$. Define

$$
\begin{aligned}
\Lambda=\bigcap_{r} \bigcap_{n}\left\{0<\zeta<\infty, X_{\zeta_{-}} \text {exists in } E, h\left(X_{\zeta^{-}}\right)=h\left(X_{\zeta}\right)_{-},\right. \\
\left.\hat{U}^{r}\left(h g_{n}\right)\left(X_{\zeta^{-}}\right)=\hat{U}^{r}\left(h g_{n}\right)\left(X_{\zeta}\right)_{-}\right\},
\end{aligned}
$$

where $r$ runs over the strictly positive rationals. Then $\Lambda \in \mathcal{F}^{0}$ and for each $t \geq 0$

$$
\theta_{t}^{-1}(\Lambda) \cap\{t<\varsigma\}=\Lambda \cap\{t<\varsigma\} .
$$

The set $\Lambda$ will play an important role in the remainder of this section. We begin with the following property, the converse of which will be proved shortly in (7.9).

(7.3) Proposition. Let $\mu \in \mathcal{M}$, and let $u=U(\mu)$. Then $P^{x / u}(\Lambda)=1$ a.e. $m$ on $E_{u}$.

Proof. Choose $g \in b \mathcal{E}^{+}$with $m(g)<\infty, \int u g d m<\infty$, and $g=0$ off $E_{u}$. We are going to apply the dual of (6.10). Here is the correspondence needed. In 
this application, $(u, \hat{U} g, g m, \mu)$ correspond to $(\hat{u}, u, \mu, \hat{\mu})$ in (the duals of $)(6.5)$ and (6.10). Note that $g m(u)=\int g u d m<\infty$. Hence, by the dual of $(6.10)$,

$$
P^{g u m / u}(\Lambda)=P^{g u m / u}(0<\varsigma<\infty) .
$$

But $P^{x / u}(\varsigma>0)=1$ for all $x \in E$, while $P^{x / u}(\varsigma<\infty)=\lim _{\alpha \rightarrow 0} E^{x / u}\left(e^{-\alpha \varsigma}\right)$. Let $u^{\alpha}=U^{\alpha}(\mu)$. Then by $(6.8)$

$$
u(x) E^{x / u}\left(e^{-\alpha \zeta}\right)=u^{\alpha}(x)
$$

except possibly on $\{u=\infty\} \cup N$ where $N$ is a finely open $m$-polar set. From (7.5), $u^{\alpha} \leq u$ a.e. $m$ and $u^{\alpha}$ increases a.e. $m$ to a function $v$ as $\alpha$ decreases to zero through rational values. Thus $v \leq u$ a.e. $m$. On the other hand if $f \geq 0, \int f u^{\alpha} d m=\mu \hat{U}^{\alpha} f$ increases to $\mu \hat{U} f=\int u f d m$ as $\alpha$ decreases to zero, and so $v=u$ a.e. $m$. Thus from (7.5), $E^{x / u}\left(e^{-\alpha \varsigma}\right)$ increases to one a.e. $m$ on $E_{u}$, and so $P^{x / u}(\varsigma<\infty)=1$ a.e. $m$ on $E_{u}$. Now (7.4) gives $P^{g u m / u}(\Lambda)=\int g u d m$, and since $g$ is arbitrary on $E_{u}$ subject to the conditions in the first sentence of this proof, we see that $P^{x / u}(\Lambda)=1$ a.e. $m$ on $E_{u}$. Q.E.D.

Since $P^{x / u}(\varsigma<\infty)=0$ if $x \notin E_{u}$ (recall our convention if $x \notin E_{u}$ ), (7.3) may be restated: If $u=U(\mu)$ with $\mu \in \mathcal{M}$, then

$$
P^{x / u}(\Lambda)=1_{E_{u}} \quad \text { a.e. } m \text {. }
$$

From (7.2) it is clear that for any $u \in S, x \rightarrow P^{x / u}(\Lambda)$ is excessive for the semigroup $\left(P_{t}^{u}\right)$. Hence there exists $w \in S$ with $w(x)=u(x) P^{x / u}(\Lambda)$ on $\{u<\infty\}$. This simple result is stated in $[\mathbf{1 0},(1.1)]$ and proved in $[\mathbf{1 1},(1.3)]$. For completeness, here is the proof. Let $\varphi(x)=P^{x / u}(\Lambda)$ with $u \in S$. Then $\varphi$ is excessive for $\left(P_{t}^{u}\right), \varphi \in \mathcal{E}$, and $\varphi=0$ off $E_{u}$. Set $\bar{w}(x)=u(x) \varphi(x)$ if $u(x)<\infty$ and $\bar{w}(x)=\infty$ if $u(x)=\infty$. Since $P_{t}(x, \cdot)$ is carried by $\{u<\infty\}$ if $u(x)<\infty$, one has for $x \in E_{u}$

$$
P_{t} \bar{w}(x)=P_{t}(u \varphi)(x)=u(x) P_{t}^{u} \varphi(x),
$$

and this increases to $\bar{w}(x)$ as $t$ decreases to zero. If $u(x)=0, P_{t} \bar{w}(x) \leq P_{t} u(x)=0$ for all $t \geq 0$. Therefore $P_{t} \bar{w}$ increases to $\bar{w}$ on $\{u<\infty\}$ as $t$ decreases to zero. Clearly $P_{t} \bar{w} \leq \bar{w}$ everywhere, and so defining $w=\lim _{t \downarrow 0} P_{t} \bar{w}, w \in S$ and $w=\varphi u$ on $\{u<\infty\}$. In particular, $w=u \varphi$ a.e. $m$ because $m(u=\infty)=0$.

The next result is a key property of $\Lambda$.

(7.7) Proposition. Let $u \in S$. Then $u(x) P^{x / u}(\Lambda)$ is the potential of a measure $\mu \in \mathcal{M}$.

REMARK. This really means the excessive function $w$ introduced above (7.7) is the potential of $\mu$. But $U(\mu)$ is only determined a.e. $m$ and $w=u(\cdot) P^{/ u}(\Lambda)$ a.e. $m$, and so the above loose statement is not unreasonable.

PROOF. We are going to apply (A14) in the Appendix to the processes $X^{u}$ and $\hat{X}$ which are in weak duality relative to $u m$. Note that $\Lambda$ defined in (7.1) agrees with $\Lambda$ defined in (A12). According to (A14) there exists a $\sigma$-finite measure $\mu$ on $E$ such that for each $\alpha \geq 0$ and $f \in \mathcal{E}^{+}$

$$
(f \mu) \hat{U}^{\alpha}=E^{\cdot / u}\left[e^{-\alpha \varsigma} f\left(X_{\varsigma^{-}}\right) ; \Lambda\right] u m,
$$

and $\mu$ is, in fact, the Revuz measure of $A_{t}=1_{\Lambda} 1_{[\varsigma, \infty[}(t)$ regarded as an additive functional charging $\varsigma$ of $X^{u}$. When $\alpha=0$ and $f=1,(7.8)$ states that $u P^{\cdot / u}(\Lambda)=$ $U(\mu)$. Note that $\mu \in \mathcal{M}$ since $u<\infty$ a.e. $m$. Q.E.D.

Here is the promised third characterization of potentials. 
(7.9) Theorem. Let $u \in S$. Then $u$ is the potential of a measure $\mu \in \mathcal{M}$ if and only if $P^{\cdot / u}(\Lambda)=1$ a.e. $m$ on $E_{u}$.

Proof. Let $w \in S$ with $w=u P^{\cdot / u}(\Lambda)$ on $\{u<\infty\}$. By $(7.7), w=U(\mu)$ with $\mu \in \mathcal{M}$. If $P^{\cdot / u}(\Lambda)=1$ a.e. $m$ on $E_{u}$, then $u=w$ a.e. $m$, and so $u=U(\mu)$. The converse was proved already in (7.3). Q.E.D.

The set $\Lambda$ enables us to give another description of the Riesz decomposition (4.5) and (4.8) of an element $u \in S$ into the potential of a measure $\mu \in \mathcal{M}$ and an element $h \in \mathcal{H}$. Given $u \in S$, then, as we have shown in $(7.7), u P^{\cdot / u}(\Lambda)=w$ is the potential $U(\mu)$ of a measure $\mu \in \mathcal{M}$. It follows from (7.2) that $x \rightarrow P^{x / u}\left(\Lambda^{c}\right)$ is excessive for $\left(P_{t}^{u}\right)$, and consequently there exists an $h \in S$ with $u P^{\cdot / u}\left(\Lambda^{c}\right)=h$ on $\{u<\infty\}$. Hence

$$
u=w+h \quad \text { on }\{u<\infty\}
$$

and, in particular a.e. $m$.

(7.11) Proposition. Let $u \in S$. Then (7.10) is the decomposition described in (4.5) and (4.8).

ProOF. By (7.7) and (7.10), $w$ is a potential of a measure in $\mathcal{M}$ and $w$ is strongly $m$-dominated (4.6) by $u$. Suppose $p=U(\nu)$ is the potential of a measure $\nu \in \mathcal{M}$ and $p$ is strongly $m$-dominated by $u$. Then there exists $r \in S$ with $u=p+r$ a.e. $m$. Using (5.3) and the remarks following (6.5) we see that a.e. $m$,

$$
u P^{\cdot / u}(\Lambda)=p P^{\cdot / p}(\Lambda)+r P^{\cdot / r}(\Lambda)=p+r P^{/ r}(\Lambda)
$$

where the second equality follows from (7.3). Thus $p$ is strongly $m$-dominated by $w=u P^{\cdot / u}(\Lambda)$ and so $w$ is the potential $U(\mu)$ in (4.8). If $u=w+h_{0}$ a.e. $m$ is the decomposition of (4.8), then because of (7.10), $h=h_{0}$ a.e. $m$, and this establishes (7.11). Q.E.D.

The formula (7.8) and the interpretation of $\mu$ as the Revuz measure of $A_{t}=$ $1_{\Lambda} 1_{[\varsigma, \infty[}(t)$ relative to $\left(X^{u}, \hat{X}, u m\right)$ enable one to write down several expressions for $\mu$. (Remember, $\mu$ is the measure such that $u P^{\cdot / u}(\Lambda)=U(\mu)$.) For example, from (A1) and (A2)

$$
\begin{aligned}
\mu(f) & =\lim _{t \downarrow 0} t^{-1} \int u(x) E^{x / u}\left[f\left(X_{\zeta-}\right) ; \Lambda, \zeta \leq t\right] m(d x) \\
& =\lim _{\alpha \rightarrow \infty} \alpha \int u(x) E^{x / u}\left[e^{-\alpha \zeta} f\left(X_{\zeta^{-}}\right) ; \Lambda\right] m(d x) .
\end{aligned}
$$

If $\left(\varphi_{n}\right) \subset b \mathcal{E}^{+}$is chosen so that $\hat{U} \varphi_{n} \uparrow 1$, then using (7.8) with $\alpha=0$ gives

$$
\mu(f)=\lim _{n} \int \varphi_{n}(x) u(x) E^{x / u}\left[f\left(X_{\zeta^{-}}\right) ; \Lambda\right] m(d x) .
$$

In particular, if there exists a $\psi_{\alpha}$ with $\hat{U}^{\alpha} \psi_{\alpha}=1$, then

$$
\mu(d y)=\int \psi_{\alpha}(x) u(x) E^{x / u}\left[e^{-\alpha \varsigma} ; X_{\varsigma^{-}} \in d y, \Lambda\right] m(d x) .
$$

When $\hat{X}$ is honest, that is, $\alpha \hat{U}^{\alpha} 1=1$ for $\alpha>0,(7.14)$ reduces to

$$
\mu(d y)=\frac{1}{\alpha} \int u(x) E^{x / u}\left[e^{-\alpha \varsigma} ; X_{\zeta-} \in d y, \Lambda\right] m(d x)
$$

provided $\alpha>0$. 
All of the formulas (7.12)-(7.15) relate $\mu$ to the distribution of $X_{\zeta-}$ on $\Lambda$ under $P^{\cdot / u}$.

Finally it is worthwhile to remark explicitly that (7.11) implies that $u P^{\cdot / u}\left(\Lambda^{c}\right) \in$ $\forall$ for each $u \in S$.

Appendix. The purpose of this Appendix is to extend some of the results of [5] about the Revuz measure of a homo ${ }^{-e n e o u s ~ r a n d o m ~ m e a s u r e ~(H R M) ~ t o ~ H R M s ~}$ which are allowed to charge $\varsigma$. All unexplained notation and terminology may be found in [5].

Suppose $X$ is a Borel right process and $m$ is a fixed ( $\sigma$-finite) excessive measure for $X$. We suppose that $t \rightarrow X_{t}$ has left limits in $E P^{m}$ a.s. on $] 0, \varsigma[$. No duality hypotheses are assumed at the moment. Let $R^{+}$be the Borel $\sigma$-field of $\mathbf{R}^{+} \equiv[0, \infty[$. Recall that a (raw) HRM, $\kappa$, is a kernel from $(\Omega, \mathcal{F})$ to $\left(\mathbf{R}^{+}, R^{+}\right)$such that $\kappa(\omega, \cdot)$ is carried by $] 0, \zeta(\omega)$ [ and is a countable sum of finite measures for almost all $\omega$, and satisfies $\kappa\left(\theta_{T} \omega, B\right)=\kappa(\omega, B+T(\omega))$ a.s. on $\{T<\infty\}$ for each stopping time $T$ and Borel set $B \in R^{+}$. If $\kappa(\omega, \cdot)$ is only carried by $\left.] 0, \zeta(\omega)\right] \cap \mathbf{R}^{+}$we say that $\kappa$ is an HRM possibly charging $\zeta$. The Revuz measure $\mu=\mu_{\kappa}$ of an HRM, $\kappa$, is defined by

$$
\mu(f)=\lim _{t \downarrow 0} t^{-1} E^{m} \int_{] 0, t]} f\left(X_{s-}\right) \kappa(d s)
$$

for $f \in \mathcal{E}^{+}$. It is well known, and an easy consequence of $[\mathbf{5},(8.5)]$, that

$$
\mu(f)=\lim _{\alpha \rightarrow \infty} \alpha E^{m} \int_{0}^{\infty} e^{-\alpha s} f\left(X_{s-}\right) \kappa(d s)
$$

for $f \in \mathcal{E}^{+}$where the limit in (A2) is an increasing limit as $\alpha$ increases to infinity. Of course, these limits may be infinite. If $\kappa$ is an HRM possibly charging $\zeta$ the limits in (A1) and (A2) make sense and exist (possibly infinite) and are equal if $f=1$ (that is, $f\left(X_{s-}\right)$ is set equal to one in (A1) and (A2)), or for any $f \in \mathcal{E}^{+}$if $X_{\zeta-}$ exists in $E$ a.s. $P^{m}$ on $\{\kappa(\{\zeta\})>0\}$. In the latter case $\mu$ is again called the Revuz measure of $\kappa$ and it is a measure on $E$.

If $X$ is a standard process and $\left.\left.A_{t}=\kappa(] 0, t\right]\right)$ is a natural additive functional with uniformly bounded jumps, then Revuz [7] has shown that $\mu_{\kappa}$ is $\sigma$-finite. For general Borel right processes his argument shows that for such a $\kappa, \mu_{\kappa}$ is a countable sum of finite measures. See Example (A3) below. If we assume that $X$ has a weak dual $\hat{X}$ with respect to $m$ and $\left.\left.A_{t}=\kappa(] 0, t\right]\right)$ is natural and finite on $] 0, \zeta[$, then it can be shown using results in [5] that $\mu_{\kappa}$ is $\sigma$-finite. In all of these assertions $\kappa$ is an HRM. If $\kappa$ is allowed to charge $\zeta$, they may fail as example (A4) below shows.

(A3) EXAMPLE. Let $\left\{\theta_{k}\right\}$ be a strictly increasing sequence in $] 0, \pi[$, and in the complex plane $\mathbf{C}$, let $R_{k}=\left\{r e^{i \theta_{k}} ; r>0\right\}$ be the open ray in the upper half plane making angle $\theta_{k}$ with the positive real axis. Let $R_{-}=\left\{r e^{-i \pi / 2} ; r \geq 1\right\}$ and define $E=\bigcup_{k>1} R_{k} \cup\{0\} \cup R_{-}$. The process $X$ is defined as follows: If $X(0) \in R_{k}$, then $X$ translates at unit speed towards the origin along $R_{k}$ and as it approaches zero it jumps to $e^{-i \pi / 2}$ and continues to translate down $R_{-}$at unit speed. If $X(0) \in R_{-}, X$ just translates down $R_{-}$at unit speed, and if $X(0)=0$, then $X$ remains at zero for an exponential holding time with parameter one and then dies. The reader should draw a sketch. It is evident that $X$ is a (transient) Borel right process having left 
limits in $E$ on $] 0, \zeta] \cap \mathbf{R}^{+}$. Let $m$ be one-dimensional Lebesgue measure on each of the rays $R_{k}, k \geq 1$, and on $R_{-}$, and let $m(\{0\})=0$. Then $m$ is excessive, but $X$ does not have a weak dual relative to $m$. Let $L=\sup \left\{t: X_{t} \in \bigcup R_{k}\right\}$ and $T=\inf \left\{t: X_{t} \in R_{-}\right\}$where, as usual, the supremum and infimum of the empty set are 0 and $+\infty$. Let $A_{t}=1_{\{0<L \leq t\}}=1_{\{0<T \leq t\}}$. Then $A$ is a predictable additive functional (not charging $\zeta$ ) with $A_{t} \leq 1$. It is easy to see that the Revuz measure $\mu$ of $A$ is given by $\mu=\infty \cdot \varepsilon_{0}$; that is, $\mu(f)=\infty$ if $f(0)>0$ and $\mu(f)=0$ if $f(0)=0$ for all $f \in \mathcal{E}^{+}$. Thus $\mu$ is not $\sigma$-finite although it is a countable sum of finite measures.

(A4) Example. We modify the previous example. This time $E=\bigcup_{k \geq 1} R_{k} \cup\{0\}$ and the process now dies as it approaches zero along $R_{k}$. (This is just the previous process killed at $T$.) With $m$ as before but restricted to this $E, X$ has a weak dual $\hat{X}$ relative to $m$. Namely if $\hat{X}(0)=0$ then $\hat{X}$ remains at zero for an exponential holding time with parameter one and then dies, and if $\hat{X}(0) \in R_{k}, \hat{X}$ translates away from the origin at unit speed along $R_{k}$. This time let $A_{t}=1_{\cup R_{k}}\left(X_{0}\right) 1_{[\varsigma, \infty[}(t)$. Note that $X_{\zeta-} \in E$. Once again $A$ is a predictable additive functional and its Revuz measure is $\infty \cdot \varepsilon_{0}$.

To begin the general discussion let $\kappa$ be an HRM of $X$ possibly charging $\zeta$. We emphasize that $\kappa(d t)$ is carried by $] 0, \zeta]$. The following results are the analogs of (8.5) and (8.7) of [5]. The proofs there need only minor changes to extend to the present situation, and so we omit them. It is not assumed that $X$ has a weak dual relative to $m$. We write $\Delta \kappa(\zeta)=\kappa(\{\zeta\})$ for the mass of $\kappa$ at $\zeta$.

(A5) THEOREM. Let $\kappa$ be an HRM of $X$ possibly charging $\zeta$. Let $\eta$ be a measure on $E$ that is a countable sum of finite measures. Then for each $\alpha \geq 0$

$$
\left.\left.\lim _{t \downarrow 0} t^{-1} E^{\eta U^{\alpha}} \kappa(] 0, t\right]\right)=E^{\eta} \int_{0}^{\infty} e^{-\alpha t} \kappa(d t) .
$$

(A6) THEOREM. Let $\kappa$ be an HRM of $X$ possibly charging $\varsigma$ and suppose that $\left.\left.\lim _{t \downarrow 0} t^{-1} E^{m} \kappa(] 0, t\right]\right)<\infty$. Let $f \in b \mathcal{E}^{*+}$ with $t \rightarrow f\left(X_{t-}\right)$ left continuous on $] 0, \zeta[$ a.s. $P^{m}$. Suppose also that a.s. $P^{m}$ on $\{\Delta \kappa(\zeta)>0\}$, one has $X_{\zeta-\text { existing in } E}$ and $f\left(X_{\zeta_{-}}\right)=f\left(X_{\zeta}\right)_{-}$. Then

$$
\left.\left.\mu(f) \equiv \lim _{t \downarrow 0} t^{-1} E^{m} \int_{j 0, t]} f\left(X_{s-}\right) \kappa(d s)=\lim _{t \downarrow 0} t^{-1} E^{m}\left[f\left(X_{0}\right) \kappa(] 0, t\right]\right)\right] .
$$

We next need the Revuz formula for HRM's which may charge $\zeta$. In the remainder of this section we suppose that $X$ and $\hat{X}$ are in weak duality relative to $m$; that is, (1.1) and (1.2) are satisfied. The proof of the next result is different from the one given in [5] for the analogous result [5, (9.3)] and seems better suited to the present situation.

(A8) THEOREM. Let $\kappa$ be an HRM possibly charging $\zeta$ with $\mu(1)=\mu_{\kappa}(1)<\infty$. Let $u_{\kappa}^{\alpha}(x)=E^{x} \int_{0}^{\infty} e^{-\alpha t} \kappa(d t)$ be the $\alpha$-potential of $\kappa$. Choose a countable collection $\left(g_{n}\right) \subset b \mathbf{C}_{u}^{+}(E)$ which is uniformly dense in $b \mathbf{C}_{u}^{+}(E)$ and with $g_{1}=1$. Define

$$
\Gamma=\bigcap_{r} \bigcap_{n}\left\{0<\varsigma<\infty, X_{\zeta_{-}} \text {exists in } E, \hat{U}^{r} g_{n}\left(X_{\zeta^{-}}\right)=\hat{U}^{r} g_{n}\left(X_{\zeta}\right)_{-}\right\} \text {, }
$$

where $r>0$ runs through the positive rationals. If $\Delta \kappa(\zeta)=1_{\Gamma} \Delta \kappa(\varsigma)$ a.s. $P^{m}$, then for each $\alpha \geq 0, \mu \hat{U}^{\alpha}=u_{\kappa}^{\alpha} m$. 
REMARKS. Of course, $u_{\kappa}^{\alpha} \in S^{\alpha}$ and so (A8) asserts in the terminology of $\S 3$ that $\mu \in \mathcal{M}^{\alpha}$ for each $\alpha \geq 0$ and $u_{\kappa}^{\alpha}=U^{\alpha}(\mu)$. We shall say that $\Delta \kappa(\zeta)$ is carried by $\Gamma$ when the condition $\Delta \kappa(\zeta)=1_{\Gamma} \Delta \kappa(\zeta)$ a.s. $P^{m}$ holds.

PROOF. Let $f=\hat{U}^{r} g_{n}$ for some $n$ and positive rational $r$. It is well known, e.g. $[5,(9.6)]$, that $t \rightarrow f\left(X_{t-}\right)$ is $P^{m}$ a.s. left continuous on $] 0, \zeta[$. Since $\Delta \kappa(\varsigma)$ is carried by $\Gamma$ we may apply (A7) to obtain

$$
\left.\left.\left.\left.\mu \hat{U}^{r} g_{n}=\lim _{t \downarrow 0} t^{-1} E^{m}\left[f\left(X_{0}\right) \kappa(] 0, t\right]\right)\right]=\lim _{t \downarrow 0} t^{-1} E^{f m}[\kappa(] 0, t]\right)\right] .
$$

If $h \in \mathcal{E}^{+}, \int f h d m=\left(h, \hat{U}^{r} g_{n}\right)=\left(U^{r} h, g_{n}\right)$, and so $f m=\left(g_{n} m\right) U^{r}$ as measures. Now using (A5)

$$
\mu \hat{U}^{r} g_{n}=E^{g_{n} m} \int_{0}^{\infty} e^{-r t} \kappa(d t)=\left(g_{n}, u_{\kappa}^{r}\right)
$$

Thus $\mu \hat{U}^{r}\left(g_{n}\right)=\left(g_{n}, u_{\kappa}^{r}\right)$ for all $n$. Since $g_{1}=1, \int u_{\kappa}^{r} d m=\mu \hat{U}^{r}(1)<\infty$. It follows from the density of $\left(g_{n}\right)$ in $b \mathbf{C}_{u}^{+}(E)$ that $\mu \hat{U}^{r}=u_{\kappa}^{r} m$ for each positive rational $r$. But as $r$ decreases to $\alpha, u_{\kappa}^{r}$ increases to $u_{\kappa}^{\alpha}$, and $\mu \hat{U}^{r}$ increases to $\mu \hat{U}^{\alpha}$, completing the proof of $(\mathrm{A} 8)$.

(A9) CoRollary. Let $F(t, x) \in\left(R^{+} \times \mathcal{E}\right)^{+}$. Under the hypotheses of (A8),

$$
\int m(d x) E^{x} \int F(t, x) \kappa(d t)=\int_{0}^{\infty} d t \int \mu \hat{P}_{t}(d x) F(t, x) .
$$

PROOF. If $F(t, x)=e^{-\alpha t} f(x)$ with $f \in b \mathcal{E}$, (A10) follows from (A8). For $F(t, x)=e^{-t} \varphi(t) f(x)$ with $\varphi$ and $f$ bounded, (A10) is of the form

$$
\int m(d x) f(x) E^{x} \int e^{-t} \varphi(t) \kappa(d t)=\int_{0}^{\infty} e^{-t} \varphi(t) \mu \hat{P}_{t}(f) d t .
$$

Both sides are bounded measures in $\varphi$ and $f$ and since equality holds for $\varphi(t)=$ $e^{-\alpha t}$, it holds for all bounded $\varphi$ and $f$. Now (A9) follows by a monotone class argument. Q.E.D.

The next formula is obtained from (A8) exactly as in the proof of $[\mathbf{5},(9.9)]$. Of course $\kappa$ is assumed to satisfy the hypotheses of (A8);

$$
(f \mu) \hat{U}^{\alpha}(d x)=\left[E^{x} \int_{0}^{\infty} e^{-\alpha t} f\left(X_{t-}\right) \kappa(d t)\right] m(d x)
$$

for $f \in \mathcal{E}^{+}$.

REMARK. (A8) was proved assuming $\mu_{\kappa}(1)<\infty$. Using the fact that for any $\mathrm{HRM}, \kappa$, possibly charging $\left.\left.\zeta, 2^{k} E^{m} \kappa(] 0,2^{-k}\right]\right)$ increases to $\mu_{\kappa}(1)$ as $k$ increases to infinity, one can extend (A8), (A9) and (A11) to any HRM, $\kappa$, possibly charging $\zeta$ with $\Delta \kappa(\varsigma)$ carried by $\Gamma$ such that $\kappa=\sum \kappa_{n}$ where $\mu_{\kappa_{n}}(1)<\infty$ for each $n$.

We now specialize the preceding results to a situation of particular importance for this paper. We assume $X$ and $\hat{X}$ are in weak duality with respect to $m$ and that $\hat{X}$ is transient. Fix $q \in \mathcal{E}$ with $0<q \leq 1, m(q)<\infty$, and $h \equiv \hat{U} q \leq 1$. Let $\left(g_{n}\right)$ be a countable, uniformly dense subset of $b \mathbf{C}_{u}^{+}(E)$ with $g_{1}=1$. Define

$$
\begin{array}{r}
\Lambda=\bigcap_{r} \bigcap_{n}\left\{0<\varsigma<\infty, X_{\zeta_{-}} \text {exists in } E, h\left(X_{\zeta^{-}}\right)=h\left(X_{\zeta}\right)_{-},\right. \\
\left.\hat{U}^{r}\left(h g_{n}\right)\left(X_{\zeta^{-}}\right)=\hat{U}^{r}\left(h g_{n}\right)\left(X_{\zeta}\right)_{-}\right\},
\end{array}
$$


where $r>0$ runs over the positive rationals. Finally define

$$
A_{t}=1_{\Lambda} 1_{[\varsigma, \infty[}(t) .
$$

Then $A$ is an (adapted) additive functional of $X$ which charges $\zeta$-in fact, $d A_{t}$ is carried by $\{\zeta\}$.

(A 14) Proposition. Let $\mu$ be the Revuz measure of $A$ defined in (A13). Then $\mu$ is a $\sigma$-finite measure on $E$ and, for each $\alpha \geq 0, f \in \mathcal{E}^{+}$,

$$
(f \mu) \hat{U}^{\alpha}(d x)=E^{x}\left[e^{-\alpha \zeta} f\left(X_{\zeta^{-}}\right) ; \Lambda\right] m(d x) .
$$

Proof. The function $h=\hat{U} q$ is coexcessive, $0<h \leq 1$, and $X$ and $\hat{X}^{h}$ are in weak duality with respect to $h m$ by (5.4). Let $\left(\hat{V}^{\alpha}\right)$ be the resolvent of $\hat{X}^{h}$ so that

$$
\hat{V}^{\alpha} f=\frac{1}{h} \hat{U}^{\alpha}(h f) \text {. }
$$

Consequently, for each $g_{n}$ and rational $r>0$, one has on $\Lambda$

$$
\hat{V}^{r} g_{n}\left(X_{\zeta^{-}}\right)=\frac{\hat{U}^{r}\left(h g_{n}\right)\left(X_{\zeta^{-}}\right)}{h\left(X_{\zeta^{-}}\right)}=\frac{\hat{U}^{r}\left(h g_{n}\right)\left(X_{\zeta}\right)_{-}}{h\left(X_{\zeta}\right)_{-}}=\hat{V}^{r} g_{n}\left(X_{\zeta}\right)_{-} .
$$

Let $\nu$ be the Revuz measure of $A$ relative to $\left(X, \hat{X}^{h}, h m\right)$ and let

$$
u_{A}^{\alpha}(x)=E^{x} \int_{0}^{\infty} e^{-\alpha t} d A_{t}
$$

be the $\alpha$-potential of $A$. Then

$$
\alpha E^{h m} \int_{0}^{\infty} e^{-\alpha t} d A_{t}=\alpha\left(h, u_{A}^{\alpha}\right)=\alpha\left(\hat{U} q, u_{A}^{\alpha}\right)=\alpha\left(q, U u_{A}^{\alpha}\right)
$$

The resolvent equations for additive functionals $[1,(\mathrm{IV}-2.3)]$ states that $u_{A}^{\alpha}+$ $\alpha U u_{A}^{\alpha}=u_{A}$, and so $\alpha U u_{A}^{\alpha} \leq u_{A} \leq 1$. Thus

$$
\alpha\left(h, u_{A}^{\alpha}\right) \leq(q, 1)=m(q)<\infty,
$$

and hence $\nu$ is finite because by (A2), the left-most member of (A16) increases to $\nu(1)$ as $\alpha$ increases to infinity. Consequently, taking (A15) into account, (A8) and (A11) may be applied to $A, \nu$ and $\left(X, \hat{X}^{h}, h m\right)$ to give, for each $\alpha \geq 0$ and $f \in \mathcal{E}^{+}$,

$$
(f \nu) \hat{V}^{\alpha}=\left(U_{A}^{\alpha} f\right) \cdot h m
$$

where

$$
U_{A}^{\alpha} f(x)=E^{x} \int_{0}^{\infty} e^{-\alpha t} f\left(X_{t-}\right) d A_{t}=E^{x}\left[e^{-\alpha \varsigma} f\left(X_{\varsigma^{-}}\right) ; \Lambda\right] .
$$

Recalling the definition of $\hat{V}^{\alpha},(\mathrm{A} 17)$ becomes

$$
\left(f \frac{\nu}{h}\right) \hat{U}^{\alpha}=U_{A}^{\alpha} f \cdot m
$$

or defining $\lambda=\nu / h,(f \lambda) \hat{U}^{\alpha}=U_{A}^{\alpha} f \cdot m$. Clearly $\lambda$ is $\sigma$-finite and we shall complete the proof of (A14) by showing $\lambda=\mu$. To this end $\mu$, the Revuz measure of $A$ relative to $(X, \hat{X}, m)$, is given by

$$
\mu(f)=\lim _{\alpha \rightarrow \infty} \alpha \int U_{A}^{\alpha} f d m=\lim _{\alpha \rightarrow \infty} \alpha(f \lambda) \hat{U}^{\alpha}(1)=\lambda(f)
$$


since $\alpha \hat{U}^{\alpha} 1$ increases to 1 as $\alpha$ increases to infinity. Q.E.D.

REMARK. In the proof of (A14) we assumed that $\hat{X}$ is transient. This can be avoided by passing to the one subprocesses $Y$ and $\hat{Y}$ of $X$ and $\hat{X}$. The processes $Y$ and $\hat{Y}$ are in weak duality relative to $m$ and $A$ may be regarded as an additive functional of $Y$ in a standard manner. It is then easy to check that the Revuz measure of $A$ relative to $(Y, \hat{Y}, m)$ is still $\mu$. We leave the standard details to the interested reader.

\section{REFERENCES}

1. R. M. Blumenthal and R. K. Getoor, Markov processes and potential theory, Academic Press, New York, 1968.

2. R. K. Getoor, Markov processes: Ray processes and right processes, Lecture Notes in Math., Vol. 446, Springer-Verlag, Berlin, Heidelberg and New York, 1975.

3. _ Transience and recurrence of Markov processes (Sém. de Prob. XIV), Lecture Notes in Math., vol. 784, Springer-Verlag, Berlin, Heidelberg and New York, 1980.

4. R. K. Getoor and J. Glover, Markov processes with identical excessive measures, Math. Z. 184 (1983), 287-300.

5. R. K. Getoor and M. J. Sharpe, Naturality, standardness, and weak duality for Markov processes, Z. Wahrsch. Verw. Gebiete (submitted).

6. G. A. Hunt, Markov processes and potentials. I, II, III, Illinois J. Math. 1 (1957), 44-93; 316-369; 2 (1958), 151-213.

7. D. Revuz, Mesures associées aux fonctionnelles additives de Markov. II, Z. Wahrsch. Verw. Gebiete 16 (1970), 336-344.

8. M. J. Sharpe, General theory of Markov processes (to appear).

9. J. B. Walsh, Markov processes and their functionals in duality. Z. Wahrsch. Verw. Gebiete 24 (1972), 229-246.

10. Math. Soc., Providence, R.I., 1976, pp. 131-152.

11. _ Notes on the duality of Markov processes, Paris lecture notes (unpublished).

Department of Mathematics, University of California, La Jolla, CaliforNIA 92093

Department of MAthematics, University of Florida, Gainesville, Florida 32611 